\title{
SCHRÖDINGER OPERATORS WITH MAGNETIC FIELDS. I. GENERAL INTERACTIONS
}

\author{
J. AVRON, I. HERBST AND B. SIMON
}

\section{\$1. Introduction}

The rigorous study of Schrödinger operators, $-\Delta+V$, has become a highly developed mathematical discipline during the past 25 years, see e.g., [39, 40, 41] for a systematic review. There has been much less study of Schrödinger operators with magnetic fields, i.e., $-(\vec{\nabla}-i \vec{a})^{2}+V$, where $\vec{a}$ is the magnetic vector potential so that the magnetic field $\vec{B}$ is given by $\vec{B}=\vec{\nabla} \times \vec{a}$ (in three dimensions; in general dimension, $\vec{B}$ should be thought of as a 2 -form). The bulk of the literature allowing magnetic fields requires $\vec{a}$ to go to zero at infinity which is quite far from the important case of constant $\vec{B}(\vec{a}$ is linear). One big exception to this is the work on essential self-adjointness which, since the paper of Ikebe-Kato [25], has placed no restriction on the behavior of $\vec{a}$ at infinity (e.g., Ikebe-Kato allow an arbitrary $\vec{a}$ which is once continuously differentiable); see $[44,50,45,66]$ for recent developments. The only previous studies of "spectral theory" in cases where $\vec{B}$ does not go to zero at infinity are those of Jörgens [27] and Schechter [44] (see also [28, 18, 56] and paper II of our series [6]) locating the essential spectrum in $N$-body problems when one mass is infinite and the potentials between particles of finite mass are positive, Grossman [21] discussing direct integral decompositions for periodic potentials in magnetic fields and Lavine-O'Carroll [34] who discuss a very special problem (to which we return in $\$ 5$ and another paper [7]). Simultaneously to our work, Combes, Schrader, and Seiler [11] have studied the semi-classical limit with results that overlap ours on one point (see \$2).

This is the first of a series of papers on the spectral and scattering theory of Schrödinger operators with magnetic fields. In this paper we study the theory for general $\vec{a}$ 's and $V$ 's and also the special case where $\vec{a}$ is linear $(\vec{B}$ constant $)$. In II of the series [6], we describe some rather novel features of the reduction of the center of mass in constant $\vec{B}$ field. In III of the series [7], we describe some special problems associated with the case $\vec{B}=$ constant, $V=$ sum of Coulomb 
potentials, which describes an atom in a magnetic field. Announcements of some of our results appear in $[4,5]$.

Basic to many of our results is the following: If $V$ is fixed and $H(\vec{a})=H_{0}(\vec{a})+$ $V \equiv-(\vec{\nabla}-i \vec{a})^{2}+V$ then

$$
\left|e^{-t H(\vec{a})} \phi\right| \leq e^{-t H(0)}|\phi|
$$

pointwise. The history of (1.1) and references for proofs can be found in $\$ 2$ where (1.1) is extensively applied. Typical of the results that follow easily from (1.1) is the one that says that the wave operators $\Omega^{ \pm}\left(H_{0}(\vec{a})+V, H_{0}(\vec{a})\right)$ exist and are complete for $V \in L^{1}\left(\mathbb{R}^{3}\right) \cap L^{2}\left(\mathbb{R}^{3}\right)$ and $\vec{a}$ " arbitrary".

Classically, the Hamiltonian function corresponding to $H(\vec{a})$ is

$$
H_{\text {class }}(\vec{a})=(\vec{p}-\vec{a})^{2}+V(\vec{r}) \text {. }
$$

Thus, for example, $\left(|\cdot| \equiv\right.$ Lebesgue measure in $\left.\mathbb{R}^{6}\right)$

$$
\left|\left\{(\vec{p}, \vec{x}) \mid H_{\text {class }}(\vec{a}) \leq E\right\}\right|
$$

is independent of $\vec{a}$ since changing $\vec{a}$ just changes positions of centers of spheres in $p$-space. This fact suggests that various bounds on numbers of bound states, etc. by classical quantities (see $\$ 2$ for references) should extend to arbitrary $\vec{a}$, and using (1.1), we will see this in $\S 2$.

In $\$ 3-\$ 5$ we concentrate on the case

$$
\vec{a}=\frac{1}{2}\left(\vec{B}_{0} \times \vec{r}\right)
$$

of constant field $\vec{B}_{0}$. Among our results, we mention those that depend on the fact that a constant field, $\vec{B}_{0}$, confines particles in the directions perpendicular to $\vec{B}_{0}$; e.g., classically the orbits are spirals coming from circles orthogonal to $\vec{B}_{0}$ and drift parallel to $\vec{B}_{0}$. Thus scattering only requires fall off for $V$ in directions parallel to $\vec{B}_{0}$. Moreover, the bound state structure should be reminiscent of one dimension rather than three; see $\$ 5$.

When $\vec{a}$ has the form of (2), it is not true that $H_{0}(\lambda \vec{a}) \rightarrow H_{0} \equiv-\Delta$ in norm resolvent sense as $\lambda \downarrow 0$; rather the convergence is only in strong resolvent sense. This means that the stability of isolated eigenvalues of $H_{0}+V$ under perturbation to $H_{0}(\lambda \vec{a})+V$ is not completely trivial. This problem is discussed in $\$ 6$.

Two magnetic potentials $\vec{a}_{1}$ and $\vec{a}_{2}$ with $\vec{a}_{1}-\vec{a}_{2}=\operatorname{grad} \lambda$ for some $\lambda \in C^{1}$ are said to be equivalent under a (smooth) gauge transformation. Since they lead to the same magnetic field, they should produce equivalent physics and indeed one has formally that

$$
U_{\lambda}\left[H_{0}\left(\vec{a}_{2}\right)+V\right] U_{\lambda}^{-1}=H_{0}\left(\vec{a}_{1}\right)+V
$$

where $\left(U_{\lambda} f\right)(\vec{x})=\exp (i \lambda(\vec{x})) f(\vec{x})$. Since $\lambda$ is $C^{\mathbf{1}}$, it is easy to make (1.3) more than formal. For example, if $H_{0}(\vec{a})+V$ is defined as a sum of forms by closing the obvious form on $D_{1}=\left\{f \in L^{2} \mid f\right.$ is $\left.C^{1}, D f \in L^{2}\right\}$ (this requires $\vec{a} \in L_{\text {loc }}^{2}$ and 
some hypothesis on $V$, e.g., $V \geq 0, V \in L_{\text {loc }}^{1}$; see $\S 2$ ), then (1.3) holds in the sense that $U_{\lambda}^{-1}$ is an isomorphism from $D\left(H\left(\vec{a}_{2}\right)\right)$ onto $D\left(H\left(\vec{a}_{1}\right)\right)$ and the operators are unitarily equivalent. In one dimension, any continuous $a$ is equivalent under a smooth gauge transformation to 0 ; take

$$
\lambda(x)=\int_{0}^{x} a\left(x^{\prime}\right) d x^{\prime} .
$$

In particular, one dimensional $\boldsymbol{H}(\boldsymbol{a})$ are not especially interesting.

We would like to next give a brief discussion of quantum mechanical magnetic bottles:

Definition. A $C^{1}$ vector potential $\vec{a}$ is called a magnetic bottle of the first (resp. second) [resp. third] kind if and only if $H_{0}(\vec{a})$ has some non-zero eigenfunction (resp. $H_{0}(\vec{a})$ has pure point spectrum) [resp. $H_{0}(\vec{a})$ has compact resolvent].

It is fairly obvious that magnetic bottles are easier to make in even dimensions than in odd dimensions because classically particles circle in planes or products of planes. For example in two dimensions, $\vec{a}$ of the form (1.2) (with $\vec{B}_{0}$ in a fictitious third dimension) is a magnetic bottle of the second kind and more generally, if $\vec{a}$ is chosen in $2 \nu$ dimensions so that $B$ is a two form of maximal rank, then $\vec{a}$ is a magnetic bottle. On the other hand $\vec{a}$ 's corresponding to constant field are never magnetic bottles in odd dimensions and there are no magnetic bottles in one dimension since any $\vec{a}$ can be gauged away.

One might think there are no magnetic bottles in any odd dimension. But, we shall see in $\S 2$, that for $\vec{B}=\left(y^{2}+z^{2}, x^{2}+z^{2}, x^{2}+y^{2}\right)$, the corresponding $\vec{a}$ is a magnetic bottle of the third kind.

It is hard to put a finger on what precisely distinguishes odd and even dimensions. In $\$ 2$ we will construct a two-dimensional $\vec{a}$ which is a magnetic bottle of the second kind with $\vec{B} \rightarrow 0$ at infinity $(\vec{a} \rightarrow \infty$ though), while this particular construction will not work in odd dimension. We thus make:

CONJECTURE B. If $\vec{a} \in C^{1}\left(\mathbb{R}^{3}\right)$ and $\vec{B}=\vec{\nabla} \times \vec{a} \rightarrow 0$ at infinity, then $\vec{a}$ is not a magnetic bottle of the second kind.

Two conjectures appear in this paper. One involves the behavior of new states that appear in constant fields for small coupling (Conj SC in §5) and the second is Conj $\mathrm{B}$ above.

It is a pleasure to thank M. Aizenman, E. B. Davies, J. Fröhlich and E. Seiler for a number of useful discussions, and L. Pitt and T. Kato for making some of their results available prior to publication.

\section{§2. Scattering and spectral theory: General $\vec{a}$}

Let $\vec{a}$ be in $L_{\text {loc }}^{2}\left(\mathbb{R}^{\nu}\right)$ and let $\Pi_{j}=$ closure of $-i \nabla_{j}-a_{j}$ on $C_{0}^{\infty}\left(\mathbb{R}^{\nu}\right)$ (it is closable since it is symmetric). By a theorem of Von Neumann, $\sum_{j=1}^{\nu} \Pi_{j}^{*} \Pi_{j}$ is a self 
adjoint operator (see e.g., [39]); we denote it by $H_{0}(\vec{a})$. In terms of quadratic forms, $H_{0}(\vec{a})$ is the operator associated with the closure of the form

$$
(\phi, \psi) \rightarrow \sum_{j} \int\left[\left(i \nabla_{j}+a_{j}\right) \phi\right](\vec{x}) \overline{\left[\left(i \nabla_{j}+a_{j}\right) \psi\right](\vec{x})} d^{\nu} x
$$

on $C_{0}^{\infty}$. This last statement is not trivial; it is proven in [66].

In a preliminary version of this manuscript, certain results for very general $\vec{a}$ 's and/or $V$ 's depended on two conjectures which have now been proven by Kato [64], Dodds and Fremlin [69], and Pitt [65], motivated, in part, by our conjectures. We begin by stating Kato's result in a slightly stronger form due to Simon [66]:

THEOREM 2.1. (Kato [64]) Let $\vec{a}_{n} \rightarrow \vec{a}$ in $L_{\text {loc }}^{2}\left(\mathbb{R}^{\nu}\right)$ (i.e., $\left\|\left(\vec{a}_{n}-\vec{a}\right) f\right\|_{2} \rightarrow 0$ for each $\left.f \in C_{0}^{\infty}\right)$. Then $H_{0}\left(\vec{a}_{n}\right) \rightarrow H_{0}(\vec{a})$ in strong resolvent sense. In particular, any $H_{0}(\vec{a})$ is the strong resolvent limit of $H_{0}\left(\vec{a}_{n}\right)$ with $\vec{a}_{n} \in C_{0}^{\infty}$.

To give the Dodds-Fremlin-Pitt result, we need:

Definition. Let $B, C$ be bounded operators on $L^{2}(M, d \mu)$. We write $B \leq C$ if and only if $|B \phi| \leq C|\phi|$ (pointwise) for any $\phi$.

Following the notation of [39], we use $\Phi_{\infty}$ to denote the ideal of compact operators and $\mathscr{I}_{p}$ to denote the operators with $\|A\|_{p}=\operatorname{Tr}\left(|A|^{p}\right)^{1 / p}<\infty$, so e.g., $\mathscr{I}_{2}$ is the Hilbert-Schmidt operators. Dodds and Fremlin [69] and Pitt [65] have independently proven:

Theorem 2.2. Let $B \leq C$ and $C \in \mathscr{I}_{\infty}$, then $B \in \mathscr{I}_{\infty}$.

Remarks 1. If $\infty$ is replaced by $p$ and $p<2$, the statement is false. If $p=2 n$ with $n$ an integer, it is an easy statement to prove. On this basis, it was conjectured in $[54,59]$ that the result holds for $\Phi_{p}, p \geq 2$, (the conjecture included $p=\infty$ ). For $p \neq 2 n, \infty$, the conjecture remains open.

2. In many places below where we use Theorem 2.2 one could avoid its use by employing devices from [3].

Basic to our results in this section will be:

THEOREM 2.3. If $\vec{a}$ is in $L_{\mathrm{loc}}^{2}$, then for any $t$ :

$$
e^{-t H_{0}(\vec{a})} \leq e^{-t H_{0}}=e^{t \triangle} \text {. }
$$

(2.1) was originally conjectured by Simon and stated in [55] with the remark that Nelson had noted that it followed easily from the Feynman-Kac-Ito formula for $e^{-t H_{0}(\vec{a})}$ in terms of Wiener path and Ito stochastic integrals. The details of this argument may be found in [60]. An alternate argument exploiting Kato's inequality can be found in $[23,57]$. In any event, $(2.1)$ is proven in $[60,23,57]$ for $\vec{a} \in C_{0}^{\infty}$ and then follows by Theorem 2.1 and a limiting argument for arbitrary $\vec{a} \in L_{\text {loc }}^{2}$. A "direct proof" for all $\vec{a} \in L_{\text {loc }}^{2}$ can be found in [66].

Typical of the applications of (2.1) are the following two results patterned on ideas of Davies [13] and Faris [19]: 
THEOREM 2.4. If $V$ is a multiplication operator which is $H_{0}$ bounded with relative bound $\alpha$, then for any $\vec{a} \in L_{\text {loc }}^{2}, V$ is $H_{0}(\vec{a})$-bounded with relative bound at most $\alpha$. If $\alpha<1$, then $H_{0}(\vec{a})+V$ is self-adjoint on $D\left(H_{0}(\vec{a})\right)$ and

$$
e^{-t\left(H_{0}(\vec{a})+V\right)} \leq e^{-t\left(H_{0}+V\right)}
$$

Remark. The first part of Theorem 2.4 is a result of Schechter (p. 168 in [44])

THEOREM 2.5. If $|V|$ is a multiplication operator which is $\boldsymbol{H}_{0}$-form bounded with relative bound $\alpha$, then for $\vec{a} \in L_{\text {loc }}^{2},|V|$ is $H_{0}(\vec{a})$-form-bounded with relative bound at most $\alpha$. If $V=V_{+}-V_{-}$with $V_{+} \in L_{10 c}^{1}$, and $V_{-} H_{0}$ form-bounded with relative bound $\alpha<1$, then $H_{0}(\vec{a})+V$ is a closable form on $C_{0}^{\infty}$ and (2.2) holds.

Proof. Since

$$
(A+E)^{-1}=\int_{0}^{\infty} e^{-E t} e^{-t A} d t
$$

(2.1) implies that $\left(H_{0}(\vec{a})+E\right)^{-1} \leftrightarrows\left(H_{0}+E\right)^{-1}$ so, for $\phi \in L^{2}$ :

$$
\begin{aligned}
\left\|V\left(H_{0}(\vec{a})+E\right)^{-1} \phi\right\| & \leq\left\|V\left(H_{0}+E\right)^{-1}|\phi|\right\| \\
& \leq\left\|V\left(H_{0}+E\right)^{-1}\right\|\|\phi\|
\end{aligned}
$$

i.e., $\left\|V\left(H_{0}(\vec{a})+E\right)^{-1}\right\| \leq\left\|V\left(H_{0}+E\right)^{-1}\right\|$. Since $\alpha=\lim _{E \rightarrow \infty}\left\|V\left(H_{0}+E\right)^{-1}\right\|$ the first part of Theorem 2.4 is proven. If $V \geq 0,(2.2)$ follows from the Trotter formula, (2.1) and the fact that $e^{-t V} \geq 0$ pointwise. The proof of the first part of Theorem 2.5 is similar using

$$
(A+E)^{-1 / 2}=\int t^{-1 / 2} e^{-t E} e^{-t A} d t / \Gamma\left(\frac{1}{2}\right) .
$$

(2.2) follows for general $V$ by letting

$$
V_{n}(\vec{x})=\left\{\begin{array}{cc}
n & V(\vec{x}) \geq n \\
V(\vec{x}) & -n \leq V(\vec{x}) \leq n \\
-n & V(\vec{x}) \leq-n
\end{array}\right.
$$

and noting that $\left(H_{0}(\vec{a})+V_{n}\right) \rightarrow H_{0}(\vec{a})+V$ in strong resolvent sense as $n \rightarrow \infty$ $[47,58]$.

(2.2) and the formula:

$$
\inf \operatorname{spec}(A)=\inf _{\phi \neq 0}\left[\lim _{t \rightarrow \infty}-t^{-1} \ln \left(\phi, e^{-t A} \phi\right)\right]
$$

imply that for any $\vec{a} \in L_{\text {loc }}^{2}$ :

$$
\text { inf spec }\left[H_{0}(\vec{a})+V\right] \geq \inf \operatorname{spec}\left[H_{0}+V\right]
$$


a result of Simon [15]. Indeed (2.1) was found by attempting to generalize (2.3) to "'finite temperature.'

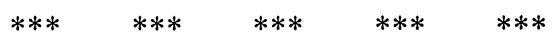

Theorem 2.6. Let $\vec{a}$ be in $L_{\mathrm{loc}}^{2}$ and $V$ a multiplication operator. If $V\left(H_{0}+E\right)^{-1}$ is compact then $V\left(H_{0}(\vec{a})+E\right)^{-1}$ is compact.

Proof. $\quad V\left(H_{0}(\vec{a})+E\right)^{-1} \leqq|V|\left(H_{0}+E\right)^{-1}$. Now use Theorem 2.2.

THEOREM 2.7. Let $V$ be a multiplication operator of the form of theorem 2.5. Suppose that $H_{0}+V$ has compact resolvent. Then $H_{0}(\vec{a})+V$ has compact resolvent for any $\vec{a} \in L_{\text {loc }}^{2}$.

Proof. Follows from (2.2) and Theorem 2.2.

Thus far, we have shown that $H_{0}(\vec{a})$ is at least as "well-behaved" as $H$; if $\vec{a}$ is big enough, it can be "better-behaved". The discussion below is related to the question of magnetic bottles; see $\$ 1$.

THEOREM 2.8. Suppose that for some function $f \rightarrow \infty$ at $\infty$ and $C$ :

$$
(\phi,|f| \phi) \leq C\left(\phi,\left(H_{0}(\vec{a})+1\right) \phi\right)
$$

for all $\phi \in C_{0}^{\infty}$. Then $H_{0}(\vec{a})$ has compact resolvent.

Proof. (2.4) says that $\left(H_{0}(\vec{a})+1\right)^{-1 / 2}|f|^{1 / 2}$ is bounded. Since $f \rightarrow \infty$ at $\infty$, $(1+|f|)^{-1 / 2}\left(H_{0}+1\right)^{-1 / 2}$ is compact [40], so by Theorem 2.2 and the bound

$$
\begin{gathered}
(1+|f(\vec{x})|)^{-1 / 2}\left(H_{0}+1\right)^{-1 / 2} \geqq(1+|f(\vec{x})|)^{-1 / 2}\left(H_{0}(\vec{a})+1\right)^{-1 / 2}, \\
(1+|f(\vec{x})|)^{-1 / 2}\left(H_{0}(\vec{a})+1\right)^{-1 / 2}
\end{gathered}
$$

is compact. Since $\left(H_{0}(\vec{a})+1\right)^{-1 / 2}(|f(\vec{x})|+1)^{1 / 2}$ is bounded, $\left(H_{0}(\vec{a})+1\right)^{-1}$ is compact.

The following is related to the standard proof of the uncertainty principle:

TheOREM 2.9. Let $\Pi_{j}=-i \nabla_{j}-a_{j}$ with $\vec{a} \in C^{1}$. Then for any $\phi \in C_{0}^{\infty}$ :

$$
\left|\left(\phi,\left(\partial_{i} a_{j}-\partial_{j} a_{i}\right) \phi\right)\right| \leq\left\|\Pi_{j} \phi\right\|^{2}+\left\|\Pi_{i} \phi\right\|^{2} .
$$

Remark. For constant field in two dimensions, this inequality is saturated as we let $\phi$ approach the ground states for $\Pi_{1}^{2}+\Pi_{2}^{2}$. Since $H_{0}(\vec{a})$ is essentially a harmonic oscillator and $\Pi_{1}$ and $\Pi_{2}$ obey the usual $p, q$ commutation relations, this should not be surprising.

Proof. Notice the formal commutation relation

$$
\left[\Pi_{i}, \Pi_{j}\right]=i\left(\partial_{i} a_{j}-\partial_{j} a_{i}\right) .
$$

Since $\vec{a} \in C^{1}$ and $\phi \in C_{0}^{\infty}$, the formal relation can be justified to yield, by the Schwarz inequality,

$$
\begin{aligned}
\left|\left(\phi,\left(\partial_{i} a_{j}-\partial_{j} a_{i}\right) \phi\right)\right| & \leq 2\left\|\Pi_{i} \phi\right\|\left\|\Pi_{j} \phi\right\| \\
& \leq\left\|\Pi_{i} \phi\right\|^{2}+\left\|\Pi_{j} \phi\right\|^{2} .
\end{aligned}
$$


CoROllaRY 2.10. Let $\vec{a}$ be an $L_{\text {loc }}^{2}$ vector potential on $\mathbb{R}^{\nu}$ and let $\Delta_{\alpha}$ be a covering of $\mathbb{R}^{\nu}$ by cubes of size L about the points $L \alpha\left(\alpha \in \mathbb{Z}^{\nu}\right)$. For each $\Delta_{\alpha}$, suppose we can choose two unit vectors $e_{\alpha}$ and $\mu_{\alpha}$ so that $B_{\alpha} \equiv \min$ $F(x)\left(e_{\alpha}, u_{\alpha}\right)$ (where $\left.F(x) \equiv \sum_{i<j}\left(\partial_{i} a_{j}-\partial_{j} a_{i}\right)(x) d x^{i} \wedge d x^{j}\right)$ approaches $\infty$ as $\alpha \rightarrow \infty$. Then $H_{0}(\vec{a})$ has compact resolvent.

Proof. Let $\eta_{\alpha}$ be a family of $C_{0}^{\infty}$ functions with the following properties: (i) $\eta_{\alpha}$ has support in the union of $\Delta_{\alpha}$ and its $3^{\nu}-1$ neighbors, (ii) $\eta_{\alpha}=1$ on $\Delta_{\alpha}$, (iii) $\eta_{\alpha}$ is the translate of $\eta_{0}$ by $L \alpha$ units, and (iv) $1 \geq \eta_{\alpha} \geq 0$. By Theorem 2.9:

$$
\begin{aligned}
B_{\alpha}\left\|\eta_{\alpha} \phi\right\|^{2} & \leq\left(\eta_{\alpha} \phi, F\left(e_{\alpha}, \mu_{\alpha}\right) \eta_{\alpha} \phi\right) \\
& \leq\left(\eta_{\alpha} \phi, H_{0}(\vec{a}) \eta_{\alpha} \phi\right) \\
& =\sum_{i=1}^{\nu}\left\|\Pi_{i} \eta_{\alpha} \phi\right\|^{2} \leq 2 \sum_{i=1}^{\nu}\left(\left\|\eta_{\alpha} \Pi_{i} \phi\right\|^{2}+C\left\|\chi_{\alpha} \phi\right\|^{2}\right)
\end{aligned}
$$

where $\chi_{\alpha}$ is the characteristic function of $\Delta_{\alpha}$ and its neighbors and we have used $\left[\Pi_{i}, \eta_{\alpha}\right]=-i \nabla_{i} \eta_{\alpha}$. Thus

$$
\begin{aligned}
\sum_{\alpha} B_{\alpha}\left\|\eta_{\alpha} \phi\right\|^{2} & \leq 23^{\nu} \sum_{i=1}^{\nu}\left(\left\|\Pi_{i} \phi\right\|^{2}+C\|\phi\|^{2}\right) \\
& \leq C_{1}\left[\left(\phi, H_{0}(\vec{a}) \phi\right)+(\phi, \phi)\right] .
\end{aligned}
$$

Theorem 2.8 now implies the result.

Remarks. 1. For example, if $B_{x}, B_{y}, B_{z} \geq 0$ (in three dimensions) and $\boldsymbol{B}_{x}+$ $B_{y}+B_{z} \rightarrow \infty$ at $\infty$, then $H_{0}(\vec{a})$ will be a magnetic bottle of the third kind.

2. One can easily modify the above to allow a $V_{-}, V_{+}$of the form of theorem 2.5 with $B_{\alpha} \rightarrow \infty$ replaced by $\tilde{B}_{\alpha}+\min _{x \in \Delta_{\alpha}} V_{+}(\vec{x}) \rightarrow \infty$

$$
\text { where } \tilde{B}_{\alpha}=\max \left(B_{\alpha}, 0\right) \text {. }
$$

3. One can arrange for $\vec{\nabla} \times \vec{B}=0$, e.g., $B_{x}=x, B_{y}=y, B_{z}=-2 z$ so that one sets up a magnetic bottle without any currents in finite regions of space (but, of course, one needs "currents at infinity").

We conclude our discussion of magnetic bottles with a two dimensional example of a magnetic bottle of the second kind with $B \rightarrow 0$ at $\infty$. As we have already explained, we don't believe that a similar phenomenon occurs in odd dimensions.

Example. Let $a_{x}=-y \rho^{-\epsilon}, a_{y}=x \rho^{-\epsilon}$ with $0<\epsilon<1$ and $\rho=\left(x^{2}+y^{2}\right)^{1 / 2}$. Then

$$
B=(2-\epsilon) \rho^{-\epsilon}
$$

and in terms of $\rho, \phi$ coordinates and $L=\frac{1}{i} \frac{\partial}{\partial \phi}$ :

$$
H_{0}(\vec{a})=-\Delta+\rho^{2-2 \epsilon}-2 \rho^{-\epsilon} L
$$


On each subspace with $L=m, H_{0}(\vec{a})$ has compact resolvent so $H_{0}(\vec{a})$ is a magnetic bottle of the second kind; it is not of the third kind since one can find $\phi_{n}$ orthonormal so that $\left(\phi_{n}, H_{0}(\vec{a}) \phi_{n}\right) \rightarrow 0$ as $n \rightarrow \infty$ : For example, let $\|\eta\|_{2}=1$ and suppose that $\eta$ lies in $C_{0}^{\infty}$. Let

$$
\eta_{\vec{y}, n}(\vec{x})=e^{\overrightarrow{i a}(\vec{y}) \cdot \vec{x}} \eta((\vec{x}-\vec{y}) / n) .
$$

Then

$$
\left\|\left(i^{-1} \vec{\nabla}-\vec{a}\right) \underset{\eta_{y, n}}{ }\right\| \leq n^{-1}\|\vec{\nabla} \eta\|+\left\|(\vec{a}-\vec{a}(\vec{y})) \eta_{y, n}\right\| .
$$

Choose $\vec{y}_{n}$ so that the $\eta_{\vec{y}_{n}, n} /\left\|\eta_{\vec{y}_{n}, n}\right\| \equiv \phi_{n}$ have disjoint support, so that

$$
\operatorname{supp} \phi_{n} \subset\left\{\vec{x}|| \vec{x}\left|\geq \frac{1}{2}\right| \vec{y}_{n} \mid\right\},
$$

and so that $n\left|\vec{y}_{n}\right|^{-\epsilon} \rightarrow 0$ as $n \rightarrow \infty$. Then since $|\vec{\nabla} \vec{a}| \leq$ const $\rho^{-\epsilon},|\vec{a}-\vec{a}(\vec{y})|$ $\leq$ (const) $n\left|\vec{y}_{n}\right|^{-\epsilon}$ on $\operatorname{supp} \eta \vec{y}_{n}, n$ so $\left(\phi_{n}, H_{0}\left(\vec{a}_{n}\right) \phi_{n}\right) \rightarrow 0$.

Next, we consider two body scattering. We concentrate primarily on the existence and completeness question for the pair $\left(H_{0}(\vec{a})+V ; H_{0}(\vec{a})\right)$, i.e., showing that asymptotically exp $\left(-i\left[H_{0}(\vec{a})+V\right] t\right)$ looks like motion in the pure magnetic field. Other than the usual $L_{\text {loc }}^{2}$ restriction, there will be no restriction on $\vec{a}$. Later $(\S 4)$, we will prove much stronger results for the case $\vec{B}=$ constant. We note that in cases where $V$ and $\vec{a}$ go to zero at infinity as $0\left(|\vec{x}|^{-1-\epsilon}\right)$, it has already been proven (see Agmon [1], Kuroda [33] or Schechter [46]) that the pairs $\left(H_{0}(\vec{a})+V, H_{0}\right)$ and $\left(H_{0}(\vec{a}), H_{0}\right)$ have complete scattering.

THEOREM 2.11. Let $\vec{a} \in L_{\text {loc }}^{2}\left(\mathbb{R}^{3}\right)$ and let

$$
V \in L^{1}\left(\mathbb{R}^{3}\right) \cap L^{3 / 2}\left(\mathbb{R}^{3}\right) .
$$

Then $\Omega^{ \pm}\left(H_{0}(\vec{a})+V, H_{0}(\vec{a})\right)$ exist and are complete.

Proof. By the Kato-Birman theory (see e.g., [40]) it suffices to show that for $E$ real and sufficiently positive $\left(H_{0}(\vec{a})+V+E\right)^{-1}-\left(H_{0}(\vec{a})+E\right)^{-1}=C$ is trace class. Since $V$ is $H_{0}(\vec{a})$ form-bounded (see Theorem 2.5 ), with relative bound 0 , it suffices to show that $A=|V|^{1 / 2}\left(H_{0}(\vec{a})+E\right)^{-1}$ is Hilbert-Schmidt (for $C=$ $\left.-A^{*}(\operatorname{sgn} V)\left[1+(\operatorname{sgn} V)|V|^{1 / 2}\left(H_{0}+E\right)^{-1}|V|^{1 / 2}\right]^{-1} A\right)$. But $|V|^{1 / 2}\left(H_{0}(\vec{a})+\right.$ $E)^{-1} \leq|V|^{1 / 2}\left(H_{0}+E\right)^{-1}$ which is Hilbert-Schmidt.

To handle the case of general $\nu$, we note:

Theorem 2.12. Let $W \in l_{1}\left(L^{2}\right)$, let $\vec{a} \in L_{\text {loc }}^{2}\left(\mathbb{R}^{\nu}\right)$ and let $m>\frac{n}{2}$. Then $W\left(H_{0}(\vec{a})+1\right)^{-m}$ is trace class.

Remark. $\quad l_{1}\left(L^{2}\right)$ is a space introduced by Birman and Solomjak [8]: Let $\Delta_{\alpha}$ be a covering of $\mathbb{I R}^{\nu}$ by the unit cubes about lattice points. Then $f \in l^{1}\left(L^{2}\right)$ if and only if

$$
\sum_{\alpha}\left(\int_{\Delta_{\alpha}}|f(\vec{x})|^{2} d x\right)^{1 / 2}<\infty
$$


so, e.g., $f \in l^{1}\left(L^{2}\right)$ if $\int\left(1+\vec{x}^{2}\right)^{k}|f(\vec{x})|^{2} d x<\infty$ for some $k>\nu / 2$.

Proof. By the closed graph theorem and the triangle inequality for $\mathscr{I}_{1}$, it suffices to show the result in case $W$ is in $L^{2}$ with support in some $\Delta_{\alpha}$ with bound independent of $\vec{a}$ [59]. In that case we write

$|W|\left(H_{0}(\vec{a})+1\right)^{-m}=\left[|W|\left(H_{0}(\vec{a})+1\right)^{-m / 2}\left(1+\vec{x}^{2}\right)^{m / 2}\right]\left[\left(1+\vec{x}^{2}\right)^{-m / 2}\left(H_{0}(\vec{a})+1\right)^{-m / 2}\right]$.

Each factor in $[\cdots]$ is dominated in $\$ sense, by the same object with $\vec{a}=0$ in which case they are Hilbert-Schmidt (see [59] for the first factor). The proof is complete.

From a result of Yafeev [62] (obtained independently, but subsequently by Reed-Simon [42]), this theorem immediately implies:

COROLlary 2.13. If $V \in l_{1}\left(L^{2}\right)$ and is $H_{0}$ form-bounded with relative bound $\alpha<1$, then for any $\vec{a} \in L_{\text {loc }}^{2}\left(\mathbb{R}^{\nu}\right)\left(\nu=\right.$ arbitrary) $\Omega^{ \pm}\left(H_{0}(\vec{a})+V, H_{0}(\vec{a})\right)$ exist and are complete.

Next, we wish to discuss bounds on the number of negative eigenvalues of $H_{0}(\vec{a})+V$ and the related [37] question of the stability of matter. Most of the results in this subsection have been obtained in [11] independently of and approximately simultaneously to our work. Define $N_{\alpha}(V ; \vec{a})$ to be the dimension of the spectral projection for $H_{0}(\vec{a})+V$ corresponding to the interval $(-\infty,-\alpha)$. Then, the Birman-Schwinger principle (see [52] for references and a general review of bounds on $N_{\alpha}(V ; 0)$ ) asserts that for $V$ a relatively compact perturbation of $H_{0}$ and $\alpha \geq 0$ :

$$
N_{\alpha}(V ; \vec{a})=\#\left\{\mathrm{ev} \text {. of } K_{\alpha}(V ; \vec{a})>1\right\}
$$

where $K_{\alpha}(V ; \vec{a})=V^{1 / 2}\left(H_{0}(\vec{a})+\alpha\right)^{-1}|V|^{1 / 2}$ with $V^{1 / 2}=|V|^{1 / 2}$ sgn $V$. Since $\left(H_{0}(\vec{a})+\alpha\right)^{-1} \leqq\left(H_{0}+\alpha\right)^{-1}$ we immediately have:

THEOREM 2.14. In three dimensions:

$$
\begin{aligned}
N_{\alpha}(V ; \vec{a}) & \leq \operatorname{Tr}\left(K_{\alpha}(V ; 0) * K_{\alpha}(V ; 0)\right) \\
& =(4 \pi)^{-2} \int\left|V_{-}(\vec{x})\right|\left|V_{-}(\vec{y})\right||\vec{x}-\vec{y}|^{-2} \exp (-\sqrt{\alpha}|\vec{x}-\vec{y}|) d x d y
\end{aligned}
$$

where $V_{-}=\min (V, 0)$.

For $\vec{a}=0$, this is a well-known bound of Birman and Schwinger. Moreover:

THEOREM 2.15. If $\nu \geq 3$ :

$$
N_{0^{-}}(V ; \vec{a}) \leq c_{\nu} \int\left|V_{-}(\vec{x})\right|^{\nu / 2} d^{\nu} x
$$

where $c_{\nu}$ is the constant obtained by Lieb [35] in case $\vec{a}=0$. (e.g., in $\nu=3$, $c_{3}=.116$ ) 
Proof. Lieb's proof [35] depends on the formula (see [60])

$$
\operatorname{Tr}(f(K(V ; \vec{a}=0)))=\int \frac{d t}{t} e^{-\alpha t} \int d \mu_{0, \vec{x}, \vec{x} ; t} g\left(\int_{0}^{t} V(\vec{\omega}(s)) d s\right)
$$

where

$$
f(x)=\int_{0}^{\infty} e^{-y} g(x y) \frac{d y}{y}
$$

and where $d \mu_{0, \vec{x}}, \vec{x} ; t$ is conditional Weiner measure. By following the proof of this formula, one easily shows that general $\vec{a}$ can be taken on the left if one adds a factor

$$
\exp \left[\frac{i}{2} \int_{0}^{t} \operatorname{div}\left(\vec{a}(\vec{\omega}(s)) d s+i \int \vec{a} \cdot d \omega\right]\right.
$$

(Ito Stochastic integral) on the right. Thus $\operatorname{Tr}\left(f\left(K_{\alpha}(V ; \vec{a})\right)\right) \leq \operatorname{Tr}\left(f\left(K_{\alpha}(V ; 0)\right)\right)$ for any $g \geq 0$. As a result, Lieb's proof goes through without change.

The bound above for $\vec{a}=0$ has been also obtained by Rosenblum [43] and Cwickel [12] (although with larger constants $c_{\nu}$ than Lieb). To push Cwickel's proof through for $\vec{a} \neq 0$, one would use an analog of Theorem 2.2 for weak- $\mathscr{I}^{p}$ spaces. Two examples show that one cannot extend the above results in various directions:

Example 1. If we consider a constant field of magnitude $B$, then $H=$ $H_{0}(\vec{a})+V$ has essential spectrum beginning only at energy $B$. Thus $H$ can have discrete spectrum in $[0, B]$. One cannot extend the above bounds to the total number of discrete eigenvalues; indeed, if $V$ is a shallow attractive square well, $H$ will have no negative eigenvalues (by the above bounds) but it will have an infinite number of eigenvalues in $[0, B]$ ! See $\S 5$.

Example 2. The bound $N_{\alpha}(V ; \vec{a}) \leq N_{\alpha}(V ; 0)$ which one might expect on the basis of the above is false! Indeed for $\alpha>0$, this follows by first order perturbation theory. For $\alpha=0^{-}$, pick $W$ radially symmetric so that $-\Delta+W$ has one $s$-wave bound state and a $p$-wave zero energy eigenvalue. In first order perturbation theory, which is a variational upper bound to $0\left(B^{2}\right)$, if we turn on a constant field, the $p$-wave splits into one negative, one positive and one zero eigenvalue. Thus, one can pick $\vec{B}_{0}$ so that $H_{0}\left(\vec{a}\left(\vec{B}_{0}\right)\right)+W$ has two negative bound states. Now take $V=(1-\epsilon) W$ for $\epsilon$ small. Then

$$
N_{0^{-}}(V ; \vec{a}) \geq 2>N_{0^{-}}(V ; 0)=1
$$

(where $N_{0^{-}}$is the number of eigenvalues in $(-\infty, 0)$ ).

From Theorem 2.14 or Theorem 2.15 (to get the improved constant) one obtains the following results of Lieb-Thirring $[37,38]$ by just mimicking their proofs: 
THEOREM 2.16. Let $\phi$ be an antisymmetric function of $N$-variables $\left(r_{i}, \sigma_{i}\right)$ $\left(\sigma_{i}= \pm 1\right)$ and let

$$
\rho(\vec{x})=\sum_{\sigma_{i}= \pm 1, i=1, \cdots, n} \int\left|\phi\left(\vec{x}, \vec{x}_{2}, \cdots, \vec{x}_{n}\right)\right|^{2} d x_{2}-d x_{n} .
$$

Then $(\nu=3)$

$$
\left(\phi, \sum_{i=1}^{N} H_{0}(\vec{a})_{r_{i}} \phi\right) \geq D \int \rho^{5 / 3}(\vec{x}) d^{3} x
$$

where $D$ is the constant of Lieb [35] (and is independent of $\vec{a}$ ).

THEOREM 2.17. Let $\mu<\infty, \tilde{M} \leq \infty$. Let

$$
\begin{aligned}
H(N, M ; \vec{a})=\sum_{i=1}^{N} H_{0}(\vec{a})_{i} / 2 \mu & +\sum_{j=1}^{M} H_{0}(-\vec{a})_{j} / 2 \tilde{M}-\sum_{i<j}\left|\vec{r}_{i}-\vec{R}_{j}\right|^{-1} \\
& +\sum_{i<j}\left|\vec{r}_{i}-\vec{r}_{j}\right|^{-1}+\sum_{i<j}\left|\vec{R}_{i}-\vec{R}_{j}\right|^{-1}
\end{aligned}
$$

Then

$$
\inf \sigma(H(N, M ; \vec{a})) \geq-C(N+M) .
$$

J. Fröhlich (private communication) has noted one beautiful consequence of Theorem 2.17: Let $\vec{A}$ be an ultraviolet cutoff radiation field:

$$
\vec{A}(\vec{x})=\sum_{\mu=1}^{2}(2 \pi)^{-3 / 2} \int_{|\vec{k}| \leq K} e^{\overrightarrow{i k} \cdot \vec{x}}\left(a^{*}(\vec{k}) \vec{e}_{\mu}(\vec{k})+a_{\mu}(-\vec{k}) \vec{e}_{\mu}(-\vec{k})\right) \frac{d^{3} k}{\sqrt{2|\vec{k}|}}
$$

and let

$$
H_{0}(\vec{A})=\int_{|\vec{k}| \leq K} \sum_{\mu=1}^{2} a_{\mu}^{*}(\vec{k}) a_{\mu}(\vec{k})|\vec{k}| d^{3} k
$$

be the usual free photon Hamiltonian. Let

$$
H_{\mathrm{rad}}\left(N, M ; \vec{a}_{0}\right)=H_{0}(\vec{A})+H\left(N, M ; \vec{A}(\vec{x})+\vec{a}_{0}(\vec{x})\right)
$$

which describes $N$ electrons and $M$ protons interacting with "non-relativistic" radiation and an unquantized background field $\vec{a}_{0}$. Then:

COROllary 2.18 .

$$
H_{\mathrm{rad}}\left(N, M ; \vec{a}_{0}\right) \geq-C(N+M) \text {. }
$$

Proof. $\quad H_{0}(\vec{A}) \geq 0$ so we only need that $H\left(N, M ; \vec{A}(\vec{x})+\vec{a}_{0}(\vec{x})\right) \geq$ $-C(N+M)$. But the components of $\vec{A}(\vec{x})$ can be simultaneously diagonalized and thus the corollary follows directly from Theorem 2.17 . 
Remark. As stated, the constants in Theorem 2.17 and Corollary 2.18 are equal to each other and to Lieb's improved value of the Lieb-Thirring constant. However, if one Wick orders the $\vec{A}(\vec{x})^{2}$ term, then the $C$ in Corollary 2.18 diverges as $K \rightarrow \infty$.

\section{§3. Homogeneous field}

Here we want to review various properties of the "free Hamiltonian", $H_{0}(\vec{a})$, associated to constant field $\vec{B}_{0}$. We prefer to work in the gauge (1.2). $H_{0}(\vec{a})$ then has the form $\left(\hat{B}_{0}=\vec{B}_{0} /\left|\vec{B}_{0}\right|\right)$

$$
H_{0}(\vec{a})=-\Delta+\frac{B_{0}^{2}}{4}\left(\hat{B}_{0} \times \vec{r}\right)^{2}-\vec{B}_{0} \cdot \vec{L}
$$

where $\vec{L}$ is the angular momentum. If $\vec{B}_{0}=\left(0,0, B_{0}\right)$, then

$$
H_{0}(\vec{a})=-\Delta+\frac{B_{0}^{2}}{4}\left(x^{2}+y^{2}\right)-B_{0} L_{z} \equiv-d^{2} / d z^{2}+H_{o s c}-B_{0} L_{z} .
$$

It is useful to introduce the creation and annihilation operators:

$$
\begin{aligned}
& a_{x}=\frac{1}{\sqrt{B_{0}}} \frac{\partial}{\partial x}+\sqrt{\frac{B_{0}}{4}} x ; a_{x}^{\dagger}=a_{x}^{*} \\
& a_{y}=\frac{1}{\sqrt{B_{0}}} \frac{\partial}{\partial y}+\sqrt{\frac{B_{0}}{4}} y ; a_{y}^{\dagger}=a_{y}^{*}
\end{aligned}
$$

$H_{o s c}$ is simple in terms of these objects, since

$$
H_{o s c}=B_{0}\left(a_{x}^{\dagger} a_{x}+a_{y}^{\dagger} a_{y}+1\right) .
$$

$L_{z}$ is not so transparent since

$$
L_{z}=i\left(a_{y}^{\dagger} a_{x}-a_{x}^{\dagger} a_{y}\right) .
$$

We therefore change variables and let

$$
a_{ \pm}=\frac{1}{\sqrt{2}}\left(a_{x} \pm i a_{y}\right) .
$$

Then $\left[a_{\sharp}^{\#}, a_{-}^{\#}\right]=0$ and $\left[a_{ \pm}, a_{ \pm}^{\ddagger}\right]=1$ (same sign) so they generate independent oscillators. Moreover:

$$
\begin{gathered}
H_{o s c}=B_{0}\left(a_{+}^{\dagger} a_{+}+a_{-}^{\dagger} a_{-}+1\right) \\
L_{z}=a_{ \pm}^{\dagger} a_{-}-a_{+}^{\dagger} a_{+}
\end{gathered}
$$

and, in particular,

$$
H_{0}(\vec{a})=-\frac{d^{2}}{d z^{2}}+B_{0}\left(2 a_{+}^{\dagger} a_{+}+1\right)
$$


Several things are obvious from (3.1)-(3.3)

(i) The ground state energy ( $\equiv \inf \operatorname{spec}\left(H_{0}(\vec{a})\right)$ ) of $H_{0}(\vec{a})$ is $B_{0}$.

(ii) $H_{0}(\vec{a})$ has an infinite degeneracy associated with the fact that $a_{-}$and $a_{-}^{\ddagger}$ commute with $H_{0}(\vec{a})$ (Landau degeneracy). In II, we show that this degeneracy is a characteristic of any system of non-zero total charge in a constant magnetic field [6].

(iii) If we fix $L_{z}=m$, then for $m<0, a_{+}^{\dagger} a_{+}$takes the values $m, m+1, \cdots$ but for $m \geq 0, a_{+}^{\dagger} a_{+}$takes the values $0,1, \cdots$ so

$$
\begin{aligned}
\inf \operatorname{spec}\left(H_{0}(\vec{a}) \backslash L_{z}=m\right) & =B_{0} \quad(m \geq 0) \\
& =B_{0}(2|m|+1)(m<0) .
\end{aligned}
$$

(iv) If $C \psi=\bar{\psi}$ then by (3.1):

$$
C H_{0}(\vec{a}) C=\frac{-d^{2}}{d z^{2}}+H_{o s c}+B_{0} L_{z}=H_{0}(\vec{a})+2 B_{0} L_{z}
$$

In particular $H_{0}(\vec{a})\left(L_{z}=m\right)$ is antiunitarily equivalent to $\left(H_{0}(\vec{a})+2 B_{0} m\right) \uparrow$ $\left(L_{z}=-m\right)$.

The Landau degeneracy, (ii) above, is extensively studied from several points of view in II of this series [6]. One is relevant to our considerations below: The operators

$$
\vec{\rho}=\frac{1}{i} \vec{\nabla}+\vec{a}
$$

commute with $H_{0}(\vec{a})$; in fact, up to factors the $x$ and $y$ components of $\vec{\rho}$ are just $i^{-1}\left(a_{-}-a_{-}^{\dagger}\right)$ and $\left(a_{-}+a_{-}^{\dagger}\right) \cdot \vec{\rho}$ has an important physical interpretation as a pseudo-momentum, i.e. an operator commuting with $H_{0}(\vec{a})$ which generates a group, $U(\vec{\alpha})=e^{\overrightarrow{i p} \cdot \vec{\alpha}}$ obeying

$$
U(\vec{\alpha}) \vec{x} U(\vec{\alpha})^{-1}=\vec{x}+\vec{\alpha}
$$

$U(\vec{\alpha})$ thus realizes the obvious translation invariance of the physics. The special feature here is that $U(\vec{\alpha})$ is not a representation on $\mathbb{R}^{3}$ but only a representation up to a factor, explicitly

$$
U(\vec{\alpha}) U\left(\vec{\alpha}^{\prime}\right)=\exp \left[\frac{i}{2} \vec{B}_{0} \cdot\left(\vec{\alpha} \times \vec{\alpha}^{\prime}\right)\right] U\left(\vec{\alpha}+\vec{\alpha}^{\prime}\right)
$$

which follows from

$$
(U(\vec{\alpha}) f)(\vec{r})=e^{\frac{i}{2}\left(\vec{B}_{0} \times \vec{r}\right) \cdot \vec{\alpha}} f(\vec{r}+\vec{\alpha})
$$


As a final result, we want to discuss the derivation of the explicit formula (essentially due to Feynman [63])

$$
\begin{gathered}
e^{-t H_{0}(\vec{a})}\left(\vec{r}, \vec{r}^{\prime}\right)=(4 \pi t)^{-1 / 2} \frac{B_{0}}{4 \pi \sinh \left(B_{0} t\right)} \exp \left(-F\left(\vec{r}, \vec{r}^{\prime} ; t\right)\right) \\
F\left(\vec{r}, \vec{r}^{\prime} ; t\right)=(4 t)^{-1}\left[\hat{B}_{0} \circ\left(\vec{r}-\vec{r}^{\prime}\right)\right]^{2}+\frac{B_{0}}{4} \operatorname{coth}\left(B_{0} t\right)\left[\hat{B}_{0} \times\left(\vec{r}-\vec{r}^{\prime}\right)\right]^{2} \\
-\frac{1}{2} i \vec{B}_{0} \circ\left(\vec{r} \times \vec{r}^{\prime}\right) .
\end{gathered}
$$

Most ways of deriving (3.5) that we know of depend on first deriving Mehler's formula for

$$
\begin{gathered}
h(\omega)=-\frac{1}{2} \frac{d^{2}}{d x^{2}}+\frac{1}{2} \omega^{2} x^{2}: \\
e^{-t h(\omega)}(x, y)=\left[\frac{\omega}{2 \pi \sinh (\omega t)}\right]^{1 / 2} \\
\exp \left[-\frac{\omega}{2 \sinh (\omega t)}\left[\cosh (\omega t)\left(x^{2}+y^{2}\right)-2 x y\right]\right] .
\end{gathered}
$$

We know various derivations of (3.6) of which we mention: (a) One using Wick ordered exponentials, due to Simon and Höegh-Krohn [61]; see esp. [20] (b) One recognizing that $f(x) g(y) \rightarrow\left(f \Omega_{0}, e^{-t h(\omega)} g \Omega_{0}\right)\left(\Omega_{0}=g d\right.$ state of $\left.h(\omega)\right)$ is a Gaussian measure of covariance matrix $(2 \omega)^{-1}\left(\begin{array}{ll}1 & a \\ a & 1\end{array}\right)$ with $a=\exp (-t \omega)$, due essentially to Doob [17], see esp. [60]. (c) One beginning with the Feynman-Kac formula with respect to Brownian motion and doing the Gaussian integral [60].

We mention 5 ways of deriving (3.5); as a preliminary step in all of them it is useful to note that the $-\frac{d^{2}}{d z^{2}}$ commutes with $H_{0}-\left(-d^{2} / d z^{2}\right)$ so we may as well consider the two dimensional case:

(a) Notice that by $(3.1), H_{0}(\vec{a}) \uparrow\left(L_{z}=0\right)=2(h(\omega) \oplus h(\omega)) \uparrow\left(L_{z}=0\right)$ with $\omega=\frac{B_{0}}{2}$. Since the idealized vector $\delta_{0}=$ delta function at 0 has $L_{z}=0$, we have that

$$
\begin{aligned}
\left(e^{-t H_{0}(\vec{a})}\right)(\vec{r}, 0) & =\left(e^{-t H_{0}(\vec{a})} \delta_{0}\right)(\vec{r}) \\
& =e^{-2 t h(\omega)}\left(r_{x}, 0\right) e^{-2 t h(\omega)}\left(r_{y}, 0\right) \\
& =\text { given by }(3.6) .
\end{aligned}
$$

Using $U(\vec{\alpha}) e^{-t H_{0}(\vec{a})} U(\vec{\alpha})^{-1}=e^{-t H_{0}(\vec{a})}$ and (3.4), one can obtain $e^{-t H_{0}(\vec{a})}(\vec{r}+$ $\vec{\alpha}, \vec{\alpha})$ in terms of $e^{-t H_{0}(\vec{a})}(\vec{r}, 0)$. This yields $(3.5)$.

(b) If $\tilde{a}=\left(-B_{0} y, 0,0\right)$, then $\nabla \times \tilde{a}=B_{0}$ so $H_{0}(\vec{a})$ and $H_{0}(\tilde{a})$ are unitarily equivalent under an explicit gauge transformation. But $H_{0}(\tilde{a})=\left(p_{x}+B_{0} y\right)^{2}+$ 
$p_{y}^{2}$, so if $p_{x}$ is diagonalized, then $H_{0}(\tilde{a})$ is a translated harmonic oscillator. Explicitly if $U_{1}=$ multiplication by $e^{-i B_{0} x y / 2}$, then

$$
H_{1} \equiv U_{1} H_{0}(\vec{a}) U_{1}^{-1}=\left(p_{x}+B_{0} y\right)^{2}+p_{y}^{2}
$$

and if $U_{2}=e^{-i p_{x} p_{y} / B_{0}}$, then

$$
H_{2} \equiv U_{2} H_{1} U_{2}^{-1}=p_{y}^{2}+B_{0}^{2} y^{2}
$$

so $e^{-t H_{2}}$ has a kernel given by Mehler's formula. Realizing $p_{x}$ using a Fourier transform, one can obtain $U_{2}^{-1} e^{-t H_{2}} U_{2}=e^{-t H_{1}}$ and then $e^{-t H_{0}(\vec{a})}$.

(c) Once one knows that $e^{-t H_{0}(\vec{a})}(x, y)$ is a Gaussian (which follows by suitable "Trotter"-like approximations, see e.g. [60]) one can find it by computing the covariance of $f(\vec{x}) g\left(\vec{r}^{\prime}\right) \rightarrow\left(f \Omega_{0}, e^{-t H_{0}(\vec{a})} g \Omega_{0}\right)$ and inverting the $4 \times 4$ matrix. (See proof (b) of Mehler's formula).

(d) An explicit evaluation in a Feynman-Kac-Ito formula, see [60].

(e) Since one can obtain $H_{0}(\vec{a})$ from $2 B_{0}\left(a_{x}^{\dagger} a_{x}+\frac{1}{2}\right)+O a_{y}^{\dagger} a_{y}$ by a Bogoliubov transformation $\left(a_{x}, a_{y}\right) \rightarrow\left(a_{+}, a_{-}\right)$, it should be possible to obtain (3.5) from (3.6) and an explicit formula for the unitary implementing the Bogoliubov transformation.

\section{§4. Scattering and spectral theory: homogeneous magnetic field}

The moral of the scattering theory in general fields $(\$ 2)$ is that magnetic fields do not make conditions for a good scattering theory any worse; the point in this section is that constant fields can make the conditions milder: indeed, since a constant field "traps" the particle in the directions perpendicular to the field, one expects that falloff will only be required in the direction parallel to the field. This is the case:

THEOREM 4.1. Let $H$ be a self-adjoint extension of $H_{0}(\vec{a})+V\left(\right.$ on $\left.C_{0}^{\infty}\right)$ with $\vec{a}=\left(-\frac{1}{2} B_{0} y, \frac{1}{2} B_{0} x, 0\right)$ and $V$ real-valued. Suppose that

$$
\int(1+|z|)^{1+\epsilon} e^{-(1-\epsilon) \frac{B_{0}}{2}\left(x^{2}+y^{2}\right)}|V(x, y, z)|^{2} d x d y d z<\infty
$$

for some $\epsilon>0$. Then $\Omega^{ \pm}\left(H, H_{0}(\vec{a})\right)$ exist.

Proof. By Cook's method [40], it suffices to show that

$$
\int_{1}^{\infty}\left\|V e^{ \pm i t H_{0}(\vec{a})} \eta\right\| d t<\infty
$$

for a total set of $\eta$. We will take $\eta(x, y, z)=\phi(x, y) \psi(z)$ with $\phi$ an eigenfunction of $H_{o s c}$ and $L_{z}($ see $\S 3)$ so that $\phi(x, y)=$ (polynomial in $x$ and $\left.y\right)\left(e^{-\frac{B_{0}}{4}\left(x^{2}+y^{2}\right)}\right)$. In particular,

$$
|\phi(x, y)|^{2} \leq \text { (const) } e^{-(1-\epsilon) \frac{B_{0}}{2}\left(x^{2}+y^{2}\right)}
$$


Since $e^{-i t H_{0}(\vec{a})}(\phi \otimes \psi)=e^{-i t E} \phi \otimes e^{-i t p_{z}^{2}} \psi$ we see that for such $\eta,(4.2)$ is equivalent to:

$$
\int_{1}^{\infty}\left\|W e^{ \pm i t p_{z}^{2}} \psi\right\|_{L^{2}(d z)} d t<\infty
$$

where

$$
W(z)=\left[\int e^{-(1-\epsilon) \frac{B_{0}}{2}\left(x^{2}+y^{2}\right)}|V|^{2} d x d y\right]^{1 / 2} .
$$

By choosing vectors $\psi \in \mathscr{S}(\mathbb{R})$ with supp $\psi$ not containing the origin, it is easily seen that (4.1) implies (4.3) for a total set of $\psi$ [24] (see also [40]).

Remarks. 1. Roughly speaking (4.1) requires that

$$
|V(x, y, z)| \leq e^{\frac{(1-\epsilon)}{4} B_{0}\left(x^{2}+y^{2}\right)}(1+|z|)^{-1-\epsilon}
$$

and thus allows considerable growth in the $x$ and $y$ directions.

2. Theorem 4.1 should be compared with results of Avron-Herbst [3] and Davies [14]. Davies notes that in the usual $\Omega^{ \pm}(-\Delta+V,-\Delta)$ situation, falloff in the $z$-direction suffices if there is no growth in the $x y$ directions. Avron-Herbst discuss the case $H_{0}=-\Delta+c z$ of constant electric field where some mild growth in the $x y$ plane is allowed but for a rather different physical reason.

3. It is not automatic that $H_{0}(\vec{a})+V$ has self-adjoint extensions since $H_{0}(\vec{a})$ does not commute with the usual complex conjugation. But if $V$ is axially symmetric (invariant under rotations in the $x y$ plane), then one can find a conjugation commuting with $H \uparrow C_{0}^{\infty}$ on each fixed $L_{z}$ subspace, so $H_{0}(\vec{a})+V$ must have equal deficiency indices.

4. The argument in remark 3 depended on $\vec{B}=$ constant and $V$ azimuthally symmetric. Other cases can be accommodated. For example, suppose that

$$
\begin{aligned}
& a_{1}\left(-x_{1}, x_{2}, x_{3}\right)=a_{1}\left(x_{1}, x_{2}, x_{3}\right), \\
& \underset{3}{\mathrm{a}}\left(-x_{1}, x_{2}, x_{3}\right)=-\underset{3}{a_{2}}\left(x_{1}, x_{2}, x_{3}\right)\left[\text { e.g., } \vec{a}=\frac{1}{2}(\vec{B} \times \vec{r}) \text { with } \vec{B}=\left(0,0, B_{0}\right)\right]
\end{aligned}
$$

and

$$
V\left(-x_{1}, x_{2}, x_{3}\right)=V\left(x_{1}, x_{2}, x_{3}\right)
$$

then

$$
(\theta \psi)\left(x_{1}, x_{2}, x_{3}\right)=\overline{\psi\left(-x_{1}, x_{2}, x_{3}\right)}
$$

obeys $\boldsymbol{\theta H}=\boldsymbol{H} \boldsymbol{\theta}$ on $C_{0}^{\infty}$ if $V \in L_{\mathrm{loc}}^{2}, \vec{\nabla} \circ \vec{a} \in L_{\mathrm{loc}}^{2}$. Other variations on the theorem, e.g., $V(-\vec{x})=V(\vec{x}), \vec{a}(-\vec{x})=-\vec{a}(\vec{x})$ are possible.

THEOREM 4.2. Let $\vec{a}=\left(-\frac{1}{2} B_{0} y, \frac{1}{2} B_{0} x, 0\right)$ and let $V$ be a function of 
$\rho=\left(x^{2}+y^{2}\right)^{1 / 2}$ and $z$ so that

(a) $V=V_{1}+V_{2}$ with $V_{1}$ uniformly locally $L^{p}$ for some $p>\frac{3}{2}$ and $V_{2} \geq$ $-c_{1}-c_{2} \rho^{2}$ with $c_{2}<\frac{1}{4} B_{0}^{2}$

(b) for each $\epsilon>0$

$$
\int d z \rho d \rho e^{-\epsilon \rho 2}|V(\rho, z)|<\infty .
$$

Then on each $\left(L_{z}=m\right)$ subspace, $H_{0}(\vec{a})+V$ is a semibounded closable quadratic form on $Q\left(H_{0}(\vec{a})\right) \cap Q(V) \cap\left(L_{z}=m\right)$. Let $H$ be the corresponding operator. Then $\Omega^{ \pm}\left(H, H_{0}(\vec{a})\right)$ exist and are complete.

Proof. Let $A_{0}=-\Delta+\frac{1}{4} B_{0}^{2} \rho^{2}$ and $A=A_{0}+V$ on $Q\left(A_{0}\right) \cap Q(V)$. Then by (a) and a result of Strichartz (see e.g., [41]) $A$ is a semibounded closed quadratic form, so restricting to $\left(L_{z}=m\right)$ subspaces, we have the first claim. Similarly, the second claim will follow if we can show that $\Omega^{ \pm}\left(A, A_{0}\right)$ exists and is complete. By Birman's theorem (Theorem XI.10 in [40]) it suffices to show that (i) $|A|^{1 / 2} e^{-A_{0}}$ and $A_{0}^{1 / 2} e^{-A}$ are bounded and (ii) $E_{I}(A) V E_{I}\left(A_{0}\right)$ is trace class for any bounded interval $I$. (Here $E_{I}(\circ)$ is a spectral projection.)

It is easy to see that both (i) and (ii) follow if $|V|^{1 / 2} e^{-A_{0}}$ and $|V|^{1 / 2} e^{-A}$ are Hilbert-Schmidt. Let $K$ be the integral kernel of $e^{-A}$ and $K_{\alpha}$ that of $e^{-A_{\alpha}}\left(A_{\alpha}=\right.$ $\left.-\Delta+\alpha \rho^{2}\right)$. Using the inequality [68]

$$
e^{-t\left(-\Delta+W_{1}+W_{2}\right)}(\vec{x}, \vec{y}), \leq\left[e^{-t\left(-\Delta+p W_{1}\right)}(\vec{x}, \vec{y})\right]^{1 / p}\left[e^{-t\left(-\Delta+p^{\prime} W_{2}\right)}(\vec{x}, \vec{y})\right]^{1 / p^{\prime}}
$$

and a result of [60] (which extends a basic result of Herbst-Sloan [68]), one finds for any $p>1$

$$
|K(\vec{x}, \vec{y})| \leq C_{P}\left|K_{\alpha(p)}(\vec{x}, \vec{y})\right|^{1 / p}
$$

where $\alpha(p)=p\left(\frac{1}{4} B_{0}^{2}-C_{2}\right)$. So taking $p=2$, we need only show that

$$
\int|V(\vec{x})|\left|K_{\alpha(2)}(\vec{x}, \vec{y})\right| d x d y<\infty .
$$

Given the explicit form of $K_{\alpha}$ (from Mehler's formula and the kernel of $e^{-t p_{z}^{2}}$, (b) yields the finiteness of the integral.

Remark. The idea that one can improve the results of the trace class theory considerably by restricting to symmetry subspaces goes back to Kuroda [32].

As a final result in the scattering theory for perturbations of (3.1) we want to prove existence and completeness of modified wave operators for the pair $\left(H_{0}(\vec{a})+\frac{\lambda}{|\vec{r}|}, H_{0}(\vec{a})\right)$. By taking advantage of the symmetry subspace we will actually be able to reduce everything to the trace class theory and Dollard's original result [16] for the pair $\left(-\Delta+\frac{\lambda}{|\vec{r}|},-\Delta\right)$ (Remark: Actually, complete- 
ness is much easier to prove in that case than as proven in [16]; for once one proves that $-\Delta+\frac{\lambda}{|\vec{r}|}$ has simple spectrum on each fixed $l$ and $m$ subspace, a general argument of Kuroda [31] (corrected in [15]) implies that completeness follows from existence).

Definition. A modified dynamics associated to $\boldsymbol{H}_{0}$ is a family of commuting unitary maps $U_{t}$ commuting with $H_{0}$ so that for each fixed $s$,

$$
s-\lim _{t \rightarrow \infty} U_{t+s} U_{t}^{*}=e^{-i s H_{0}}
$$

If

$$
\Omega_{\bar{U}}^{ \pm}\left(H_{0}+V, H_{0}\right)=s-\lim _{t \rightarrow \pm \infty} e^{+i t\left(H_{0}+V\right)} U_{t} P_{a c}\left(H_{0}\right)
$$

exists, we say $\Omega_{U}^{ \pm}$exist and if $\operatorname{Ran} \Omega_{U}^{ \pm}=\operatorname{Ran} \Omega_{U}^{-}=\operatorname{Ran} P_{a c}\left(H_{0}+V\right)$ we say that $\Omega_{U}^{ \pm}$are complete.

Note that these definitions are slightly more general than in the usual Dollard theory [16] where $U$ is a function of $H_{0}$. In Hörmander's paper [24], $H_{0}$ is a constant coefficient pseudodifferential operator and $U_{t}$ is required to be a function of $-i \nabla$. In the above definition it might happen that $\Omega_{U}^{ \pm}$and $\Omega_{W}^{ \pm}$exist for $U$ and $W$ which were not mutually commuting. In any event, for its spectral significance, the above definition certainly suffices, i.e. existence and completeness imply that $\left(H_{0}+V\right) \uparrow \operatorname{Ran} P_{a c}\left(H_{0}+V\right)$ is unitarily equivalent to $H_{0} \uparrow \operatorname{Ran}$ $P_{a c}\left(H_{0}\right)$. Moreover, in the case below, it is not hard to show that $U_{t} \psi$ and $e^{-i t H_{0}} \psi$ asymptotically have the same $x$-space probability distribution so that the usual [16] physical interpretation of $\Omega_{\bar{U}}^{ \pm}$is possible.

THEOREM 4.3. For any $\lambda$ and $B_{0}$ there exists a modified dynamics, $U$, associated to $H_{0}(\vec{a})$ so that $\Omega_{U}^{ \pm}\left(H_{0}(\vec{a})+\lambda|\vec{r}|^{-1}, H_{0}(\vec{a})\right)$ exist and are complete.

Proof. By the composition law for wave operators if $\Omega^{ \pm}\left(H_{0}+V, H_{0}+W\right)$ and $\Omega_{\bar{U}}^{ \pm}\left(H_{0}+W, H_{0}\right)$ exist and are complete, then $\Omega_{U}^{ \pm}\left(H_{0}+V, H_{0}\right)$ exist and are complete. By a simple extension of Theorem $4.2, \Omega^{ \pm}\left(H_{0}(\vec{a})+\lambda /\left[\vec{r} \mid, H_{0}(\vec{a})+\right.\right.$ $\lambda /(|z|+1))$ exist and are complete since

$$
\int d z\left|\left(z^{2}+x^{2}+y^{2}\right)^{-1 / 2}-(|z|+1)^{-1}\right| \leq c\left|\log \left(x^{2}+y^{2}\right)\right| \cdot
$$

Thus, it suffices to prove that

$$
\Omega_{U}^{ \pm}\left(H_{0}(\vec{a})+\lambda(|z|+1)^{-1}, H_{0}(\vec{a})\right)
$$

exist and are complete for some $U$.

Next note that since $H_{0}(\vec{a})+d^{2} / d z^{2}$ has the form $A \otimes I$ where $A$ has discrete spectrum under $L^{2}\left(R^{3}\right)=L^{2}\left(R^{2}\right) \otimes L^{2}(d z),\left(H_{0}(\vec{a})+\lambda(|z|+1)^{-1}, H_{0}(\vec{a})\right)$ is a direct sum of pairs

$$
\left(-\frac{d^{2}}{d z^{2}}+C+\lambda(|z|+1)^{-1},-\frac{d^{2}}{d z^{2}}+C\right)
$$


so it suffices that

$$
\Omega_{U}^{ \pm}\left(-\frac{d^{2}}{d z^{2}}+\lambda(|z|+1)^{-1},-\frac{d^{2}}{d z^{2}}\right)
$$

exist and are complete for $U$ a function of $-d^{2} / d z^{2}$ (then, the $U$ for the full problem will be a function of $-d^{2} / d z^{2}$ and $\left.H_{0}(\vec{a})+d^{2} / d z^{2}\right)$.

By mimicking Dollard's basic method, [16], if

$$
U_{t}=e^{-i t p_{z}^{2}} \exp \left(-i \int_{0}^{t} \lambda\left(\left|2 p_{z} s\right|+1\right)^{-1} d s\right)
$$

then $\Omega_{U}^{ \pm}$exist and intertwine

$$
h=-\frac{d^{2}}{d z^{2}}+\lambda(|z|+1)^{-1} \text { and } h_{0}=-\frac{d^{2}}{d z^{2}} .
$$

Moreover, $\Omega_{U}^{ \pm}$leave the space of even (resp. odd) functions invariant, so by a theorem of Kuroda [31] (corrected in [15]), it suffices to show that $h$ has simple absolutely continuous spectrum when restricted to the even or to the odd functions.

One could presumably develop an eigenfunction expansion to prove the simplicity of the spectrum in question, but we provide a proof based on the fact that Dollard [16] has proven completeness in the three dimensional case, $V(\vec{r})=$ $|\vec{r}|^{-1}$ and therefore also for $V(\vec{r})=(|\vec{r}|+1)^{-1}\left(\right.$ since $\Omega^{ \pm}\left(-\Delta+(|\vec{r}|+1)^{-1}\right.$, $-\Delta+|\vec{r}|^{-1}$ exist and are complete by an argument of Kuroda [32]). Restricting to the $l=0$ subspace we see that

$$
\Omega_{\bar{U}}^{ \pm}\left(-\frac{d^{2}}{d r^{2}}+(r+1)^{-1},-\frac{d^{2}}{d r^{2}}\right)
$$

exists where $-\frac{d^{2}}{d r^{2}}$ has $u(0)=0$ boundary conditions on $(0, \infty)$. This pair is unitarily equivalent to the pair on the whole line restricted to the odd subspace, so simplicity is proven on the odd subspace. Since changing the half-line problem from $u(0)=0$ to $\frac{\partial u}{\partial r}(0)=0$ boundary conditions is a rank one change in the resolvents, we have completeness in that case and therefore simplicity on the even subspace.

Remark. By the usual methods, once this result is proven, one has the same result for $\lambda|\vec{r}|^{-1}+V$ where $V$ obeys the hypothesis of Theorem 4.2.

THEOREM 4.4. Let $H_{0}(\vec{a})$ be given by (3.1) with $B_{0} \neq 0$. Suppose that $V$ is a function of $\vec{r} \cdot \vec{B}_{0}$ and $\overrightarrow{\mid r} \times \vec{B}_{0} \mid$ which is locally $L^{2}$, and so that

$$
\frac{1}{4}\left|\vec{r} \times \vec{B}_{0}\right|^{2}+V
$$

is bounded from below and approaches infinity at infinity. Then $H=H_{0}(\vec{a})+V$ is essentially self-adjoint on $C_{0}^{\infty}$ and has a complete set of eigenvectors. 
Proof. Without loss take $\vec{B}_{0}=\left(0,0,\left|\vec{B}_{0}\right|\right)$. Under the hypothesis, the theorem holds by known results $[39,41]$ for $-\Delta+\frac{1}{4}\left|\vec{r} \times \vec{B}_{0}\right|^{2}+V=\tilde{H}$. But $H$ and $\tilde{H}$ leave each $L_{z}=m$ space invariant and $H \uparrow\left(L_{z}=m\right)=\tilde{H} \uparrow\left(L_{z}=m\right)-m$ so the result follows for $H$.

Remark. It can happen that Theorem 4.4 is applicable but that $H_{0}(\vec{a})+V$ isn't bounded from below (e.g., $\left.V=\left(\vec{r} \cdot \vec{B}_{0}\right)^{2}-\frac{1}{8}\left(\vec{r} \times \vec{B}_{0}\right)^{2}\right)$ or that $H_{0}(\vec{a})+V$ doesn't have discrete spectrum (e.g. $\left.V=\left|\vec{r} \cdot \vec{B}_{0}\right|\right)$.

Next, we want to show how the method of Agmon [1]-Kuroda [33] (in Agmon's version) extends to yield the absence of singular continuous spectrum for $H_{0}(\vec{a})+V$ and a large class of $V$ 's. As a by-product, one obtains a new proof of existence and completeness of wave operators. In distinction to the case of constant electric field where some new estimates are required [22], no new estimates are needed here; indeed only one dimensional estimates of a simpler nature than those in the general Agmon theory are required.

THEOREM 4.5 Let $V$ be a measurable function on $R^{3}$ so that $(1+|z|)^{1+\epsilon} V$ $\epsilon L^{2}+L^{\infty}$ for some $\epsilon>0$. Let $H_{0}(\vec{a})$ be given by (3.1) with $B_{0}$ parallel to $z$ and non-zero. Suppose that: (Hypothesis $F)(1+|z|)^{1+\epsilon} V$ is a relatively compact perturbation of $\mathrm{H}_{0}(\vec{a})$. Then:

(a) $\mathrm{H}_{0}(\vec{a})+V$ has empty singular continuous spectrum.

(b) $\Omega^{ \pm}\left(H_{0}(\vec{a})+V, H_{0}(\vec{a})\right)$ exist and are complete.

THEOREM 4.6. If $V$ is symmetric under rotations about the z-axis then hypothesis $F$ can be dropped from Theorem 4.5 .

Before going to the detailed proofs, we make a series of preliminary remarks:

(1) In following Agmon's method, we use a trick of Davies [67] to avoid the need of proving that the eigenvalues of $H_{0}(\vec{a})+V$ lie in a set with a discrete family of limit points. In fact all we show is that they lie in a closed countable set. We do not see how to use the Agmon analysis to show that the eigenvalues are discrete since $\left\{\psi \mid\left\|(1+|z|)^{\delta} \psi\right\|<\infty\left\|H_{0} \psi\right\|<B\right\}$ is not compact but in the context of theorem 4.6, we can show this on each $\left(L_{z}=m\right)$ subspace. Davies' trick allows one to improve the result of [22] to remove the hypothesis about the eigenvalues having a countable closure; it follows from the other hypotheses of that paper.

(2) It suffices to prove that there is a countable closed set $\mathscr{E}$ so that for any closed interval $[a, b]$ disjoint from $\mathscr{E}$ and any $\delta>\frac{1}{2}$ :

$$
\sup _{\epsilon>0, a \leq \mu \leq b}\left\|(1+|z|)^{-\delta}\left(H_{0}(\vec{a})+V-\mu-i \epsilon\right)^{-1}(1+|z|)^{-\delta}\right\|<\infty
$$


for then the absence of singular continuous spectrum follows from a general principle (see e.g., [41], §XIII.6) and existence and completeness of wave operators follows from the theory of local smoothness (see e.g., [41], §XIII.7 and 8). In the azimuthally symmetric case, it suffices to find a set $\mathscr{E}_{m}$ which is closed and countable so that the analog of (4.5) holds for each $H_{0}(\vec{a})+V \uparrow\left(L_{z}=m\right)$ for $[a, b] \cap \mathscr{E}_{m}=\varnothing$.

(3) For simplicity, we will suppose that $|V(\vec{r})| \leq C(1+|z|)^{-1-\epsilon}$. The local $L^{2}$ singularities can be accommodated easily as in [41].

The proofs of Theorems 4.5 and 4.6 depend on the weighted spaces $M_{\delta}=\left\{f \mid(1+|z|)^{\delta} f \in L^{2}\right\}$ with the obvious norm and the use of a simple eigentransform for $H_{0}(\vec{a})$. In terms of $a_{ \pm}$of $\S 3$, let $\phi_{n, m}$ be the (unique up to phase) function with $\left(a_{+} a_{+}\right) \phi=n \phi,\left(a_{-}^{\ddagger} a_{-}-a_{+}^{\dagger} a_{+}\right) \phi=m \phi$, i.e., $n=0,1,2, \cdots$, $m=-n,-n+1, \cdots$ Define the unitary map ${ }^{\wedge}$ from $L^{2}\left(R^{3}\right)$ to $\bigoplus_{m, n} L^{2}(R)$ by $\hat{f}(m, n, k)=(2 \pi)^{-1 / 2} \int e^{-i k z} \bar{\phi}_{m, n}(x, y) f(x, y, z) d x d y d z$

so that

$$
H_{0}(\vec{a}) f(m, n, k)=\left[B_{0}(2 n+1)+k^{2}\right] \hat{f}(m, n, k)
$$

The point is that for $\delta>\frac{1}{2}$, each function $f \in M_{\delta}$ has $\int\left|\int \bar{\phi}_{m, n} f d x d y\right|$ $d z<\infty$ (by the Schwarz inequality) so that $\hat{f}(m, n, k)$ is continuous in $k$. Moreover, by general theorems (see e.g., [39], §IX.9) specialized to one dimension, if $E_{0} \neq B_{0}(2 n+1)$ for any $n$ and

$$
\hat{f}(m, n, k)=0 ; f \in M_{\delta}\left(\delta>\frac{1}{2}\right)
$$

for all $k, n$ so that $B_{0}(2 n+1)+k^{2}=E_{0}$, then $\left(H_{0}(\vec{a})-E_{0}\right)^{-1} f$ exists as a function in $M_{\delta-1-\epsilon}$ for any $\epsilon>0$ with an inequality on the norms uniform for $E$ in compacts in the allowed region. One uses here the fact that

$$
\int(1+|z|)^{2 \delta}|f(x, y, z)|^{2} d x d y d z=\sum_{m, n} \int(1+|z|)^{2 \delta}\left|\int f \bar{\phi}_{m, n} d x d y\right|^{2} d z
$$

With these preliminaries one need only mimic the Agmon theory: To get Theorem 4.5, we note that $\left(H_{0}(\vec{a})-E \pm i 0\right)^{-1}$ exists as a map from $M_{\delta}$ to $M_{-\delta}$ for $E \neq(2 n+1) B_{0}$, and that $V\left(H_{0}(\vec{a})-E \pm i 0\right)^{-1}$ is compact (by hypothesis $F$ ) and norm continuous (in $E$ ) from $M_{\delta}$ to $M_{\delta}$. The norm continuity shows that $1+V\left(H_{0}(\vec{a})-E \pm i 0\right)^{-1}$ is invertible for $E \notin \mathscr{E}_{0}, \mathscr{C}_{0}$ a closed subset of $\left(B_{0}, \infty\right) \backslash$ $\left\{(2 n+1) B_{0}\right\}$. By the "Agmon bootstrap", any point in $\mathscr{E}$ is an eigenvalue of $H_{0}(\vec{a})+V$ so that $\mathscr{E}=\left\{(2 n+1) B_{0}\right\} \cup \mathscr{E}_{0}$ is closed and countable. (This is Davies' trick [67]). Moreover, for $E \notin \mathscr{E}$,

$$
\left(H_{0}(\vec{a})+V-E \pm i 0\right)^{-1}=\left(H_{0}(\vec{a})-E \pm i 0\right)^{-1}\left(1+V\left(H_{0}(\vec{a})-E \pm i 0\right)^{-1}\right)^{-1}
$$


is bounded from $M_{\delta}$ to $M_{-\delta}$ giving us the estimate we need. To get Theorem 4.6 we proceed similarly except we look at fixed values of $m \cdot V\left(H_{0}(\vec{a})-E \pm i 0\right)^{-1}$ is then automatically compact.

Finally, we discuss the applicability of dilation analytic ideas to the case of constant magnetic field. We deal here with the case $H_{0}(\vec{a})+V$. We hope to return to the $N$-body case (which will require the analysis of [6] on removing the center of mass) in part III of this series.

THEOREM 4.7. Let $V$ be an axially symmetric function on $R^{3}$ which is dilation analytic (as an operator perturbation of $-\Delta$ in the usual sense $[2,41]$ ) in a strip $|\operatorname{Im} \theta|<\theta_{0}$. Suppose that $V(\theta)(-\Delta+1)^{-1} \in \mathscr{I}_{\infty}$ for all $|\operatorname{Im} \theta|<\theta_{0}$. Then the resolvent of $U(\theta)\left[H_{0}(\vec{a})+V\right] U(\theta)^{-1}=H(\theta)$ has an analytic continuation from $\theta$ real to $|\operatorname{Im} \theta|<\min \left(\theta_{0}, \frac{\pi}{4}\right)$ and defines an operator $H(\theta)$ in this region. Moreover, $\sigma(H(\theta))$ is discrete outside of the set

$$
\bigcup_{n=0}^{\infty}\left\{(2 n+1) B_{0}+e^{-2 \theta} \lambda \mid \lambda \in[0, \infty]\right\}
$$

which is contained in $\sigma(H(\theta))$. In particular:

(a) $H_{0}(\vec{a})+V$ has empty singular continuous spectrum

(b) The only possible cluster points for the point spectrum of $H_{0}(\vec{a})+V$ are the numbers $(2 n+1) B_{0}$.

Proof. (a) and (b) follow from the spectral information on $H(\theta)$ in the usual way. $V(\theta)=U(\theta) V U(\theta)^{-1}$ is a multiplication operator which is $-\Delta$-compact by hypothesis. Thus, by Theorem $2.6, V(\theta)$ is $H_{0}(\vec{a})$-compact. If we can show that on each $L_{z}=m$ subspace $H_{0}(\vec{a}, \theta) \equiv U(\theta) H_{0}(\vec{a}) U(\theta)^{-1}$ is an analytic family of type (A) and

$$
\sigma\left(H_{0}(\vec{a})\right)=\bigcup_{n=\max (0,-m)}^{\infty}\left\{(2 n+1) B_{0}+e^{-2 \theta} \lambda \mid \lambda \in[0, \infty)\right\},
$$

then the desired result will follow from Weyl's theorem (see [41], §XIII.4). But for $\theta$ real

$$
U(\theta) H_{0}(\vec{a}) U(\theta)^{-1}=e^{-2 \theta}(-\Delta)+\frac{B_{0}^{2}}{4} e^{2 \theta}\left(\hat{B}_{0} \times \vec{r}\right)^{2}-\vec{B}_{0} \cdot \vec{L}
$$

so we need only note that $e^{-2 \theta} p^{2}+e^{2 \theta} x^{2}$ in one dimension is a closed operator on $D\left(p^{2}\right) \cap D\left(x^{2}\right)$ so long as $|\operatorname{Arg} \theta|<\frac{\pi}{4}$ with $\theta$ invariant spectrum (see e.g., [49]). Thus

$$
\begin{gathered}
\sigma\left(H_{o s c}(\theta)\right)=\left\{(2 n+1) B_{0} \mid n \geq 0, n+m \geq 0\right\} \\
\sigma\left(p_{z}^{2}(\theta)\right)=\left\{e^{-2 \theta} \lambda \mid \lambda \geq 0\right\}
\end{gathered}
$$


so, by Ichinose's lemma (441], §XIII.9) we have proven that

$$
\sigma\left(H_{0}(\vec{a}, \theta)\right)=\bigcup_{n=\max (0,-m)}^{\infty}\left\{(2 n+1) B_{0}+e^{-2 \theta} \lambda\right\}
$$

\section{§5. Enhanced binding}

There is an important qualitative difference between the bound state (eigenvalue) structure of $-\Delta$ in dimension $\nu \geq 3$ and in dimension $\nu \leq 2$ : For if $\nu \geq 3$, $-\Delta+\lambda V$ will have no bound states if $\lambda$ is small and $V$ is short range whereas, if $\nu \leq 2$ and $V \leq 0,-\Delta+\lambda V$ will have a bound state for any $\lambda>0$. Explicitly, $-\Delta+V$ has no bound state if $\nu \geq 3$ if $\int|V|^{\nu / 2} d^{\nu} x$ is sufficiently small [12,35, 43], while in $\nu \leq 2$ dimensions, $-\Delta+V$ has a bound state if $\int V(\vec{x}) d^{\nu} x \leq 0$, $V \not \equiv 0$ and $\int(1+|\vec{x}|)|V(\vec{x})| d x<\infty([53,10,30])(\nu=1)$ or $\int|V(\vec{x})|^{1+\epsilon} d^{2} x<$ $\infty(\nu=2)$.

As we have seen quite often already, the operator $H_{0}(\vec{a})$ of $(3.1)$ is much closer to a one dimensional Laplacian than to a three dimensional Laplacian and this is reflected in the ease with which bound states occur. Typical of the results we will prove in this section is:

THEOREM 5.1. Suppose that $V$ is azimuthally symmetric, non-positive (not identically zero) and in $L^{2}+\left(L_{\epsilon}^{\infty}\right)$ on $R^{3}$. Then for any $B_{0} \neq 0, H=H_{0}(\vec{a})+V$ has an infinite discrete spectrum and an infinity of eigenvalues in the essential spectrum $\left[B_{0}, \infty\right)$.

Remarks. 1. Note that $V$ 's of compact support are allowed so that the behavior is very different from that occurring for $-\Delta+V$.

2. The embedded eigenvalues occur for reasons of symmetry: namely for $m<0, H \uparrow\left(L_{z}=m\right)$ has essential spectrum $\left[(2|m|+1) B_{0}, \infty\right]$ so that discrete eigenvalues of $H \uparrow\left(L_{z}=m\right)$ can be in the essential spectrum of $H$. Presumably for nonsymmetric $V$ 's these eigenvalues will not occur. But they indicate the difficulty of handling embedded eigenvalues.

3 . This theorem has multiparticle analogs, see $[5,7]$.

Notice it can happen that the $V$ of Theorem 5.1 is such that $-\Delta+V$ has no eigenvalues. In that case (2.3) says that all the discrete eigenvalues of $H_{0}(\vec{a})+$ $V$ must lie in the interval $\left[0, B_{0}\right]$. We see an interesting phenomenon in that case: while inf $\operatorname{spec}\left(H_{0}(\vec{a})+V\right)$ goes up when $B_{0}$ is "turned on", so does the "binding energy", $B_{0}$-inf spec $\left(H_{0}(\vec{a})+V\right)$. In fact, Lieb has proven:

Theorem A.1. For any $V \in C_{0}^{\infty}\left(R^{3}\right)$,

$$
\inf \operatorname{spec}\left(H_{0}(\vec{a})+V\right) \leq\left|B_{0}\right|+\inf \operatorname{spec}(-\Delta+V)
$$


We give Lieb's proof in the appendix. In [7], we will prove that the binding energy is monotone increasing in $B_{0}$ in case $V=-\lambda|\vec{r}|^{-1}$.

LemMa 5.2. Let $W \in L^{1}+\left(L^{\infty}\right)_{\epsilon}$ on $(-\infty, \infty)$. Then $-\frac{d^{2}}{d x^{2}}+$ Whas a negative eigenvalue if any of the following holds:

(a) $W \leq 0, W \not \equiv 0$

(b) $\int|W(x)| d x<\infty, \int W(x) d x<0$

(c) $\int W_{+}(x) d x<\infty, \int W_{-}(x) d x=\infty$ where $W_{ \pm}=\max (0, \pm W)$.

Proof. Most of these results follow from [10, 30, 53] but a direct proof is possible. By the $L^{1}+\left(L^{\infty}\right)_{\epsilon}$ hypothesis, $-\frac{d^{2}}{d x^{2}}+W$ has essential spectrum $[0, \infty)$ (see e.g., [41]) so it suffices to find $\phi$ with $\int|\phi|^{2}<\infty, \int\left|\phi^{\prime}\right|^{2}+\int W|\phi|^{2}$ $<0$. By increasing $W$, we can suppose we are in case (b). Let $\phi_{a}(x)=e^{-a|x|}$. Then $\phi_{a}$ is piecewise $C^{1}$ so

$$
\int\left|\phi_{a}^{\prime}\right|^{2} d x=a^{2} \int e^{-2 a|x|} d x=a
$$

while

$$
\lim _{a \rightarrow 0} \int\left|\phi_{a}(x)\right|^{2} W(x) d x=\int W(x) d x<0
$$

by the dominated convergence theorem. Thus $\int\left|\phi_{a}^{\prime}\right|^{2}+\int\left|\phi_{a}\right|^{2} W<0$ for some
$a$.

Proof of Theorem 5.1. Let $m \geq 0$ and let $H_{m}=H \uparrow\left(L_{z}=m\right)$ and $\tilde{H}_{0, m}=$ $H_{o s c}-B_{0} L_{z} \uparrow\left(L_{z}=m\right)$. Let $\tilde{\phi}_{m}(\rho, \phi)=\phi_{m}(\rho) e^{i m \phi}(2 \pi)^{-1 / 2}$ be the ground state of $\tilde{H}_{0, m}$ so that $\tilde{H}_{0, m} \tilde{\phi}_{m}=B_{0} \tilde{\phi}_{m}$. Let $\psi(\rho, z)=\tilde{\phi}_{m}(\rho) \psi(z)$. Then

$$
\left(\Psi, H_{m} \Psi\right)=B_{0}+\left(\psi, h_{m} \psi\right)
$$

with

$$
h_{m}=-\frac{d^{2}}{d x^{2}}+V_{m}(z) ; V_{m}(z)=\int\left|\phi_{m}(\rho)\right|^{2} V(\rho, z) d^{2} \rho
$$

Since $h_{m}$ has a negative eigenvalue by Lemma 5.2, we can find $\psi$ with $\left(\Psi, H_{m} \psi\right)<B_{0}$. Thus, since $\sigma_{e s s}\left(H_{m}\right)=\left[B_{0}, \infty\right), H_{m}$ has an eigenvalue $E_{m}$. Since $\sigma_{\text {ess }}(H)=\left[B_{0}, \infty\right)$, the $E_{m}$ 's must approach $B_{0}$ as $m \rightarrow \infty$, and, in particular, there must be infinitely many distinct $E_{m}$.

By (2.3), $E_{m} \geq e_{0}=$ inf $\operatorname{spec}(-\Delta+V)$. Since $H \uparrow L_{z}=-m$ is unitarily equivalent to $H_{m}+2 m\left|B_{0}\right|$ (for $m>0$ ), $H$ has eigenvalues at the points $E_{m}+2 m\left|B_{0}\right|$. For $m$ sufficiently large, these numbers must be in $\left[B_{0}, \infty\right.$ ) (since $\left.E_{m}+2 m\left|B_{0}\right| \geq e_{0}+2 m\left|B_{0}\right|\right)$. 
Theorem 5.3. Suppose that $V$ is in $L^{2}+\left(L^{\infty}\right)_{\epsilon}$ on $R^{3}$ and that either:

(a) $\int_{\left|x^{2}+y^{2}\right| \leq R_{0}}|V(x, y, z)| d x d y d z<\infty$ and $V(x, y, z)<0$ for $x^{2}+y^{2} \geq$ $R_{0}$ for some $R_{0}$ or

(b) $\int|V(\vec{r})| d^{3} r<\infty, \int V(\vec{r}) d^{3} r<0$.

Then $H_{0}(\vec{a})+V$ has at least one eigenvalue in $\left(-\infty,\left|B_{0}\right|\right)$.

Remark. The proof will show that in case (a), there are infinitely many eigenvalues in $\left(-\infty,\left|B_{0}\right|\right)$ if $V$ is also azimuthally symmetric.

Proof. Let $\tilde{\phi}_{m}$ be as in the last proof and let $\Psi=\tilde{\phi}_{m}(\rho) \psi(z)$. Then for $m \geq 0$;

$$
(\Psi, H \Psi)=B_{0}+\left(\psi, h_{m} \psi\right) .
$$

Since $\phi_{m}(\rho)=c_{m}\left(B_{0}\right) \rho^{m} e^{-B_{0} \rho^{2 / 4}}$, we have that

$$
V_{m}(z)=c_{m}\left(B_{0}\right)^{2} \int V(x, y, z) \rho^{2 m} e^{-B_{0} \rho 2 / 2} d^{2} \rho
$$

Thus, in case (b)

$$
\sum c_{m}^{-2}\left(\frac{B_{0}}{2}\right)^{m}(m !)^{-1} \int V_{m}(z) d z=\int V(x, y, z) d x d y d z
$$

by the dominated convergence theorem, so some $\int V_{m}(z) d z<0$ and thus, by lemma 5.1 , some $\left(\psi, h_{m} \psi\right)<0$. In case (a), $\int V_{m}(z) d z<0$ for all sufficiently large $m$.

In [53], a machine is developed to find the ground state energy of $-\frac{d^{2}}{d x^{2}}+\lambda V$ for small $\lambda$. The above suggests this machine should be applicable to $H_{0}(\vec{a})+\lambda V$ and this is our next goal. We only work to first order although as in [53], one can obtain information in higher order.

TheOREM 5.4. Let $V \in L^{2}+\left(L^{\infty}\right)_{\epsilon}$ be azimuthally symmetric on $R^{3}$ and let $B_{0}>0$ and $m \geq 0$ be fixed. Let $|V|_{m}$ be given by (5.2) with $V$ replaced by $|V|$. Suppose that $\int(1+|z|)|V|_{m}(z) d z<\infty$ and that $\int V_{m}(z) d z<0$. Then, for all small positive $\lambda, H_{0}(\vec{a})+\lambda V \uparrow\left(L_{z}=m\right)$ has a unique eigenvalue $e_{m}(\lambda)$ in the interval $\left(-\infty, B_{0}\right)$. Moreover

$$
e_{m}(\lambda)=B_{0}-\left(\frac{\lambda}{2} \int V_{m}(z) d z+0\left(\lambda^{2}\right)\right)^{2} .
$$

Proof. The proof is patterned after that in [53] as modified in [10]. Let $H_{0, m}(\vec{a})=H_{0}(\vec{a})$ i $\left(L_{z}=m\right)$ with $m \geq 0$

$$
K(E)=V^{1 / 2}\left(H_{0, m}(\vec{a})-E\right)^{-1}|V|^{1 / 2}
$$


where $V^{1 / 2}=V /|V|^{1 / 2}$. Then as in the case of $-\Delta[53], E$ is an eigenvalue of $H_{0}(\vec{a})+V \uparrow\left(L_{z}=m\right)$ in $\left(-\infty, B_{0}\right)$ if and only if $\lambda K(E)$ has eigenvalue -1 . Let $\left|\phi_{m}><\phi_{m}\right|$ denote the operator

$$
\eta \rightarrow e^{i m \phi} \overline{\phi_{m}(\rho)} \int \phi_{m}(\rho) e^{-i m \phi} \eta \rho d \rho\left(\frac{d \phi}{2 \pi}\right)
$$

and write

$$
K(E)=A(E)+B(E)
$$

where

$$
\begin{gathered}
A(E)=V^{1 / 2}\left(1-\mid \phi_{m}><\phi_{m}\right)\left(H_{0}-E\right)^{-1}|V|^{1 / 2} \\
B(E)=V^{1 / 2}\left|\phi_{m}><\phi_{m}\right|\left(H_{0}-E\right)^{-1}|V|^{1 / 2} .
\end{gathered}
$$

Since $\operatorname{spec}\left(H_{0}\left(1-\left|\phi_{m}><\phi_{m}\right|\right)\right)=\left[3 B_{0}, \infty\right)$, we have that for $E<B_{0}$ (operator inequality)

$$
\left(1-\left|\phi_{m}><\phi_{m}\right|\right)\left(H_{0}-E\right)^{-1} \leq \frac{3}{2}\left(1-\left|\phi_{m}><\phi_{m}\right|\right)\left(H_{0}\right)^{-1} \leq \frac{3}{2} H_{0}^{-1}
$$

so that

$$
|A(E)| \leq \frac{3}{2}|V|^{1 / 2} H_{0}^{-1}|V|^{1 / 2}
$$

is uniformly bounded (in $E$ ) since $V$ is $H_{0}(\vec{a})$-bounded and $0 \notin \operatorname{spec}\left(H_{0}(\vec{a})\right.$ ).

Next note that $H_{0} \uparrow \operatorname{Ran}\left|\phi_{m}><\phi_{m}\right|=p_{z}^{2}+B_{0}$ so $B(E)=V^{1 / 2}\left|\phi_{m}><\phi_{m}\right|$ $g_{0}\left(E-B_{0}\right)|V|^{1 / 2}$ where $g_{0}(\alpha)=\left(p_{z}^{2}-\alpha\right)^{-1}$. Let $g_{1}(\alpha)$ be $\left(\tilde{p}_{z}^{2}-\alpha\right)^{-1}$ where $\tilde{p}_{z}^{2}$ is $\tilde{p}_{z}^{2}$ with Dirichlet boundary conditions and let $g_{2}=g_{0}-g_{1}$. Then [10] $g_{2}(\alpha)$ is rank 1 (in the $z$ variables) with integral kernel $e^{\left.-v-\alpha\left(|z|+\left|z^{\prime}\right|\right)\right)}(2 \sqrt{-\alpha})^{-1}$ and $g_{1}$ has a kernel dominated (pointwise) by $|z|^{1 / 2}\left|z^{\prime}\right|^{1 / 2}$ for all $\alpha \leq 0$. Write

$$
B(E)=C(E)+D(E)
$$

with $C(E)=V^{1 / 2}\left|\phi_{m}><\phi_{m}\right| g_{1}\left(E-B_{0}\right)|V|^{1 / 2}, D(E)=V^{1 / 2}\left|\phi_{m}><\phi_{m}\right|$ $g_{2}\left(E-B_{0}\right)|V|^{1 / 2}$. Then we claim that $C(E)$ is uniformly bounded, for its HilbertSchmidt norm is dominated by

$$
\int\left|z z^{\prime}\right||V(z, \rho)| \mid V\left(z^{\prime},\left.\rho^{\prime}|| \phi_{m}(\rho)\right|^{2}\left|\phi_{m}\left(\rho^{\prime}\right)\right|^{2} d^{2} \rho d^{2} \rho^{\prime} d z d z^{\prime}\right.
$$

which is finite by the hypothesis on $V_{m}$. Thus for $\lambda$ small and all $E<B_{0}$, $\lambda\|A(E)+C(E)\|<1$ so

$$
1+\lambda K(E)=[1+\lambda(A(E)+C(E))]\left(1+\lambda[\cdots]^{-1} B(E)\right)
$$

and thus $\lambda K(E)$ has eigenvalue -1 if and only if the rank 1 operator $\lambda[\cdots]^{-1} B(E)$ has eigenvalue -1 . From this point onwards, the proof follows that in [53] or [10] word for word. 
Remarks. 1. By mimicking the arguments in [10], one can easily show that if $\int(1+|z|)^{\epsilon}|V|_{m}(z)<\infty$, and $\int V_{m}<0$ then $\left(H_{0}(\vec{a})+V\right) \uparrow\left(L_{z}=m\right)$ has only one eigenvalue of order $B_{0}+0\left(\lambda^{2}\right)$ as $\lambda \rightarrow 0$ (it can have others of order $\left.B_{0}+o\left(\lambda^{2}\right)\right)$ and it is given by

$$
B_{0}-\left(\frac{\lambda}{2} \int V_{m}(z) d z\right)^{2}+o\left(\lambda^{2}\right) .
$$

2. The above reduces the analysis of the ground state on $\left(L_{z}=m\right)$ for small $\lambda$ to that of $p_{z}^{2}+\lambda V_{m}$. By extending the arguments in [10] we will show in III of this series [7] how to treat the case $V_{m}=0\left(|z|^{-1}\right)$ at infinity and thereby obtain the asymptotics of $H_{0}(\vec{a})-|\vec{r}|^{-1}$ for small $\lambda$. By scaling, this will also give us the asymptotics of $H_{0}(\vec{a})-|\vec{r}|^{-1}$ for $B_{0} \rightarrow \infty$.

COROLlaRY 5.5. There exist $B_{0} \neq 0$ and an azimuthally symmetric $V_{m}$ in $\mathscr{S}\left(R^{3}\right)$ so that the lowest eigenvalue of $H_{0}(\vec{a})+V$ does not have $L_{z}=0$.

Remarks. 1. This result is of interest because when $B_{0}=0$, general principles (e.g., [41], §XIII.12) assert that the ground state is positive and so has $L_{z}=0$.

2. Examples of this type have been constructed by different means in Lavine-O'Carroll [34].

3. One of the deepest results in this series is one in III that asserts for $V=-\lambda|\vec{r}|-1$, the ground state does have $L_{z}=0$.

Proof. Since we have claimed no uniformity in $m$ in Theorem 5.4, we cannot assert that for small $\lambda$, the ground state has that $m$ with $-\int V_{m}(z) d z$ largest but certainly if we find some $m>0$ with

$$
-\int V_{m}(z) d z>-\int V_{0}(z) d z>0
$$

then for $\lambda$ small, the ground state cannot have $L_{z}=0$, since there is a lower state with $L_{z}=m$ ! Let $W(\rho)=-\int V(\rho, z) d z$. Then (5.4) is equivalent to

$$
\int W(\rho)\left|\phi_{m}(\rho)\right|^{2}>\int W(\rho)\left|\phi_{m=0}(\rho)\right|^{2}>0 .
$$

Clearly, we can find $W(\rho)$ in $\mathscr{S}\left(R^{2}\right)$ so that (5.5) holds. Thus it suffices to show that the map $V \rightarrow W$ takes $\mathscr{S}_{a}\left(R^{3}\right)$ onto $\mathscr{S}_{a}\left(R^{2}\right)$ where $\mathscr{S}_{a}=\{f \in \mathscr{Y} \mid f$ is azimuthally symmetric $\}$. In terms of Fourier transforms, the map from $V$ to $W$ is

$$
\hat{W}(k)=-(2 \pi)^{1 / 2} \hat{V}(|k|, 0) .
$$

Given any azimuthally symmetric $W$, we can define $\hat{V}\left(k_{1}, k_{2}, k_{3}\right)=-(2 \pi)^{-1 / 2}$ $\hat{W}(|k|)$ and thereby demonstrate that the map is onto. 
Having considered the ground state energy of $H_{0}(\vec{a})+\lambda V$ for $B_{0}$ fixed and $\lambda$ small, we next turn to $\lambda$ fixed and $B_{0}$ small or $B_{0}$ large.

Theorem 5.6. Suppose that $V \in L^{2}+\left(L^{\infty}\right)_{\epsilon}$ and that $-\Delta+V$ has a discrete simple eigenvalue, $E_{0}$, with eigenvector $\psi$. Suppose that, for $B_{0}$ small, $H_{0}(\vec{a})+V$ has a unique eigenvalue $E\left(B_{0}\right)$ near $E_{0}$ (in the precise sense of stability defined in \$6). Then

$$
E\left(B_{0}\right)=E_{0}+0\left(\left|B_{0}\right|^{2}\right) \text { as } \quad B_{0} \rightarrow 0 .
$$

Remarks. 1. The question of when stability holds is discussed in $\S 6$.

2. For degenerate eigenvalues, this result is no longer true but one can prove that if $P$ is the spectral projection for $E_{0}$, then $E\left(B_{0}\right)=E_{0}-B_{0} a_{j}+0\left(B_{0}^{2}\right)$ where $a_{j}$ are the eigenvalues of $P L_{z} P$ on $P \mathscr{H}$.

3. In particular, for $B_{0}$ small and $E_{0}$ the ground state, $E\left(B_{0}\right) \leq E_{0}+\left|B_{0}\right|$ in agreement with (5.1).

Proof. Write $H_{0}(\vec{a})+V=A(\lambda)$ where $A(\lambda)=A_{0}+B(\lambda)$ and $B(\lambda)=-\lambda L_{z}+$ $\frac{1}{4} \lambda^{2} \rho^{2}$. Let $P_{\lambda}$ be the eigenprojection for $E(\lambda)$ so that $P_{\lambda} \rightarrow(\psi, \cdot) \psi$ in norm. In particular, for $\lambda$ small

$$
E(\lambda)=E_{0}+\left(\psi, P_{\lambda} \psi\right)^{-1}\left(\psi, B(\lambda) P_{\lambda} \psi\right)
$$

By general principles ([41], §XIII.11), $\psi \in D\left(r^{2}\right)$ and so $\psi \in D\left(L_{z}\right)$ since e.g.,

$$
\left\|x p_{y} \phi\right\| \leq \frac{1}{2}\left(\left\|x^{2} \phi\right\|+\left\|p_{y}^{2} \phi\right\|\right) \leq \frac{1}{2}\left(\|\Delta \phi\|+\left\|r^{2} \phi\right\|\right) .
$$

In the first place, this means that for $E \notin \sigma(A(\lambda)) \cup \sigma(A(0))$

$$
\left\|\left[(A(0)-E)^{-1}-(A(\lambda)-E)^{-1}\right] \psi\right\| \leq\left\|(A(\lambda)-E)^{-1}\right\|\left(E-E_{0}\right)^{-1}\|B(\lambda) \psi\|
$$

so that $\left\|P_{\lambda} \phi-\phi\right\|=0(\lambda)$ by the assumed stability and the formula for spectral projections as contour integrals of resolvents. Moreover, it implies that $B(\lambda) \psi=-\lambda L_{z} \psi+0\left(\lambda^{2}\right)=0(\lambda)$ and $\left(\psi, P_{\lambda} \psi\right)=1+0(\lambda)$ so

$$
E(\lambda)=E_{0}-\lambda\left(\psi, L_{z} \psi\right)+0\left(\lambda^{2}\right) .
$$

But, as $E_{0}$ is simple, $\psi=\bar{\psi}$ and thus, $\left(\psi, L_{z} \psi\right)$ is both purely real and purely imaginary; hence zero.

THEOREM 5.7. Let $V \in L^{2}+\left(L^{\infty}\right)_{\epsilon}$ be azimuthally symmetric and obey $\int \rho^{2 m}|V| d^{2} \rho d z<\infty$ for some $m \geq 0$. Suppose $\int \rho^{2 m} V(\rho, z) \rho d \rho d z<0$. Then, $H_{0}(\vec{a})+V \uparrow\left(L_{z}=m\right)$ has an eigenvalue $e_{m}\left(B_{0}\right)$ for all small $B_{0}$ and

$$
e_{m}\left(B_{0}\right) \leq B_{0}-c\left(B_{0}^{2}\right)^{m+1}
$$

for some $c>0$.

Proof. We just make explicit the argument in Theorem 5.1. Let $\psi$ be the trial function $(m \geq 0)$

$$
\psi(\rho, \phi, z)=\left(2^{m} m !\right)^{-1 / 2} B_{0}^{(m+1) / 2} \rho^{m} e^{-B_{0} \rho 2 / 4} \alpha^{1 / 2} e^{-\alpha|z|} e^{i m \phi}(2 \pi)^{-1 / 2}
$$


Then

$$
\begin{aligned}
& \left(\psi, H_{0}(\vec{a}) \psi\right)=B_{0}+\alpha^{2} \\
& (\psi, V \psi) \leq-2 \alpha B_{0}^{m+1} c
\end{aligned}
$$

for some $c>0$ and all small $B_{0}$ and $\alpha$. Choosing $\alpha=c B_{0}^{m+1}$, (5.5) results from the variational principle.

We believe that $\left(B_{0}^{2}\right)^{m+1}$ is the precise small $B_{0}$ behavior, in fact:

CONJECTURE SC. Suppose in addition to the hypotheses of Theorem 5.7, that $H_{0}+V \uparrow\left(L_{z}=m\right)$ has no bound states. Then:

$$
\lim _{B_{0} \rightarrow 0}\left[B_{0}-e_{m}\left(B_{0}\right)\right] B_{0}^{-2(m+1)}=a_{m}
$$

where

$$
a_{m}=\left[\frac{1}{2}\left(2^{m} m !\right)^{-1}\left[\int \rho^{2 m} V(\rho, z) \rho d \rho d z\right]\right]^{2} .
$$

Remarks. 1. The conjecture says that to leading order, $B_{0}-e_{m}\left(B_{0}\right)$ is given by the binding energy of $p_{z}^{2}+V_{m}(z)$ and then interchanges a limit of $\lambda \rightarrow 0$ and $e^{-B_{0} \rho^{2 / 2}} \rightarrow 1$ in the small coupling limit $\left(\frac{1}{2} \int V_{m}(z) d z\right)^{2}[53]$ for this energy.

2. If $\int \rho^{2 m}|V| d^{2} \rho d z=\infty$, and say, $V \leq 0$, there will still be a bound state obeying (5.5) but in fact $e_{m}\left(B_{0}\right)$ will be smaller then the right side of (5.5). For example if $V(\rho, z)=-(1+|\vec{r}|)^{-3-2 m}$, then the above proof shows that

$$
e_{m}\left(B_{0}\right) \leq B_{0}-d\left(B_{0}^{m+1} \ln B_{0}\right)^{2} .
$$

For some $d>0$. We believe also that this is the exact behavior.

Finally, we consider large $B_{0}$ :

THEOREM 5.8. Let $V$ be a continuous function on $R^{3}$ going to zero at infinity. Let $E\left(B_{0}\right)=\inf \operatorname{spec}\left(H_{0}(\vec{a})+V\right)$. Then

$$
\begin{aligned}
\lim _{B_{0} \rightarrow \infty} E\left(B_{0}\right)-B_{0} & =\inf \operatorname{spec}\left(p_{z}^{2}+V\right) \\
& =\min _{x, y}\left[\inf \operatorname{spec}\left(-\frac{d^{2}}{d z^{2}}+V(x, y, \cdot)\right)\right] .
\end{aligned}
$$

Proof. Since $H_{0}(\vec{a})-p_{z}^{2} \geq B_{0}$, the bound

$$
E\left(B_{0}\right) \geq B_{0}+\text { inf } \operatorname{spec}\left(p_{z}^{2}+V\right)
$$

holds for all $V$ and all $B_{0}$ so the lim half of (5.6) is trivial. Since $\sigma_{\text {ess }}\left(H_{0}(\vec{a})+V\right)$ $=\left[B_{0}, \infty\right)$, the other half of $(5.6)$ is trivial if inf $\operatorname{spec}\left(p_{z}^{2}+V\right)=0$ so we will suppose inf $\operatorname{spec}\left(p_{z}^{2}+V\right)=a<0$. Let

$$
f(x, y)=\inf \operatorname{spec}\left(-\frac{d^{2}}{d z^{2}}+V(x, y, \cdot)\right] \text {. }
$$


Then $f$ is continuous and goes to zero as $x, y \rightarrow \infty$ so we can find $x_{0}, y_{0}$ with $f\left(x_{0}, y_{0}\right)=a$. By translation covariance in the $x, y$ plane we can suppose without loss that $\left(x_{0}, y_{0}\right)=(0,0)$. Since

$$
\sigma_{e s s}\left(-\frac{d^{2}}{d z^{2}}+V(0,0, \cdot)\right]=[0, \infty)
$$

there is a $\eta(z)$ with

$$
\left[\frac{-d^{2}}{d z^{2}}+V(0,0, z)\right] \eta=a \eta
$$

Let $\Psi=\phi_{m=0}(x, y) \eta(z)$ where, as usual, $\phi_{m=0}$ is the ground state with $m=0$ of the oscillator part of $H_{0}(\vec{a})$. Then by a variational calculation with $\Psi$

$$
E\left(B_{0}\right) \leq B_{0}+a+\int|\eta(z)|^{2}\left[V_{m=0}(z)-V(0,0, z)\right] d z .
$$

Since $V$ is continuous, and $\left|\phi_{m}=0\right|^{2} \rightarrow \delta^{(2)}(x, y)$, the two dimensional $\delta$-function, as $B_{0} \rightarrow \infty$, the last term has a zero limit by the dominated convergence theorem.

Remarks. 1. In particular, this result says that the inequality in (5.1) is strict for large $B_{0}$ except for the trivial case where $E\left(B_{0}\right)=B_{0}$ for all $B_{0}$.

2. A similar argument shows that if $V$ is azimuthally symmetric and if $m$ is fixed, positive and $E_{m}\left(B_{0}\right)=\inf \operatorname{spec}\left(\left(H_{0}(\vec{a})+V\right) \uparrow L_{z}=m\right)$, then

$$
\lim _{B_{0} \rightarrow \infty} E_{m}\left(B_{0}\right)-B_{0}=\inf \operatorname{spec}\left(-\frac{d^{2}}{d z^{2}}+V(0,0, z)\right] \text {. }
$$

In particular, if $V(0,0, z) \geq 0$ but $V\left(x_{0}, y_{0}, z\right)<0$ for some $x_{0}, y_{0}$, then it can happen that $E_{m=0}\left(B_{0}\right)$ fails to obey (5.1). This shows that (5.1) cannot be proved by a perturbation argument. In the Coulomb case $E_{m=0}=E$ and a perturbation argument works (although the perturbation parameter is the Coulomb coupling constant rather than $B_{0}$ !) [7].

3. This theorem is easily extended to the case where $V_{\alpha}=\max (V,-\alpha)$ is continuous for each $\alpha>0$ even if inf $\operatorname{spec}\left(p_{z}^{2}+V\right)=-\infty$. In particular, in the case of the Coulomb potential, $E\left(B_{0}\right)-B_{0} \rightarrow-\infty$ as $B_{0} \rightarrow \infty$. We will show in [7] that the divergence is as $\left(\ln B_{0}\right)^{2}$.

\section{§6. Stability of eigenvalues}

In his study of asymptotic perturbation theory, Kato [29] singled out the notion of stability of eigenvalues:

Definition. Let $T_{n}$ be a family of closed operators thought of as a perturbation of a closed operator $T_{0}$. Let $\lambda_{0}$ be an isolated eigenvalue of $T_{0}$ of finite multiplicity $q$. Then $\lambda_{0}$ is said to be stable under the perturbation $T_{n}$ if and only if

(1) For some $\epsilon$, and any $\lambda \in\left\{\lambda|0<| \lambda-\lambda_{0} \mid<\epsilon\right\}, \lambda \in \operatorname{spec}\left(T_{n}\right)$ for all large $n$ and $\left(T_{n}-\lambda\right)^{-1} \rightarrow(T-\lambda)^{-1}$ strongly

(2) $\operatorname{dim} \operatorname{ran}\left(-(2 \pi i)^{-1} \oint_{\left|\lambda-\lambda_{\theta}\right|=-\frac{\epsilon}{2}}\left(T_{n}-\lambda\right)^{-1} d \lambda\right)=q$ 
for $n$ sufficiently large.

Remarks. 1. Condition (1) implies that for large $n$, the whole circle $\left|\lambda-\lambda_{0}\right|=\frac{\epsilon}{2}$ lies in the resolvent set of $T_{n}$, see [29].

$$
\text { 2. } P_{n}=-(2 \pi i)^{-1} \int_{\left|\lambda-\lambda_{0}\right|=\frac{\epsilon}{2}}\left(T_{n}-\lambda\right)^{-1} d \lambda
$$

is the projection onto all eigenvectors and generalized eigenvectors for $T_{n}$ with eigenvalue in the circle $([29,41])$. Under the condition of stability, it follows that $P_{n} \rightarrow P$ in norm, see [29].

3. If $T_{n}$ is replaced by a family $T(\lambda)$ indexed by small reals or small complex numbers with $|\arg \lambda| \leq \theta_{0}$, we modify the definition in the obvious way.

In this section, we are interested in stability of eigenvalues of $-\Delta+V$ under the perturbation $H_{0}(\vec{a})+V$ for $B_{0}$ small. We are interested in this not only for its own sake as a natural question but also because we have certain applications in mind in [7], especially to Borel summability of the ground state energy of atoms. Here we consider only the one body case; consideration of $n$-body problems is left to [7], but because of the interest in Borel summability we deal with the possibility of complex $B_{0}$.

It appears necessary to develop a new method for proving stability in order to handle complex $B_{0}$ and also to handle real $B_{0}$ without restricting to a fixed $L_{z}$ subspace. One previous method, applicable to anharmonic oscillators [49], is to prove norm resolvent convergence of $T_{n}$ to $T$. However this fails here (see Theorem 6.3). Two other methods [41, 49] work for positive perturbations of a self-adjoint operator for any eigenvalue below the essential spectrum: these can be used to accommodate $H_{0}(\vec{a})+V$ if we fix $L_{z}$ and deal only with real $B_{0}$. Our strategy here is to use the resolvent equations $\left(H_{0}=-\Delta\right)$ :

$$
\begin{gathered}
\left(H_{0}(\vec{a})+V-E\right)^{-1}=\left(H_{0}(\vec{a})-E\right)^{-1}\left(1+V\left(H_{0}(\vec{a})-E\right)^{-1}\right)^{-1} \\
\left(H_{0}+V-E\right)^{-1}=\left(H_{0}-E\right)^{-1}\left(1+V\left(H_{0}-E\right)^{-1}\right)^{-1} .
\end{gathered}
$$

The resolvents with $V$ fail to converge in norm; as we shall see this is due to the failure of the resolvents without $V$ to convergence in norm. There is no apriori reason that $V\left(H_{0}(\vec{a})-E\right)^{-1}$ cannot converge to $V\left(H_{0}-E\right)^{-1}$ in norm and this is precisely what we will prove. Condition (1) will then follow immediately and condition (2) will follow by further arguments.

Our main results are the following:

Definition. $\rho^{-2} L^{2}+L_{\epsilon}^{\infty}$ consists of those functions $V$ with the property that for any $\epsilon>0, V$ can be written as $V_{1}+V_{2}$ with $\left\|V_{2}\right\|_{\infty} \leq \epsilon$ and $\left(\rho^{2}+1\right) V_{1} \epsilon$ $L^{2}\left(\mathbb{R}^{3}\right)$ (with $\left.\rho=\left(x^{2}+y^{2}\right)^{1 / 2}\right)$.

For example, any function going to zero at $\infty$ lies in $\rho^{-2} L^{2}+L_{\epsilon}^{\infty}$.

Theorem 6.1. Let $V \in \rho^{-2} L^{2}+L_{\epsilon}^{\infty}$ (real-valued) and let $H_{0}(\lambda)$ be $H_{0}(\vec{a})$ with $B_{0}=(0,0, \lambda)$. Then any negative eigenvalue of $-\Delta+V$ is stable as $\lambda \rightarrow 0$ $\left(\lambda\right.$ real) under the perturbation $H_{0}(\lambda)+V$.

TheOREM 6.2. Let $V \in \rho^{-2} L^{2}+L_{\epsilon}^{\infty}$ be azimuthally symmetric but not necessarily real-valued. Let $H_{0}(\lambda)$ be as above with $|\arg \lambda| \leq \theta_{0}<\frac{\pi}{2}$. Fix m. Then 
any eigenvalve $e_{0}$, of $(-\Delta+V) \uparrow\left(L_{z}=m\right)$ with $\left|\arg e_{0}\right|>2 \theta_{0}$ is stable as $\lambda \rightarrow 0\left(|\arg \lambda| \leq \theta_{0}\right)$ under the perturbation $H_{0}(\lambda)+V \uparrow\left(L_{z}=m\right)$.

Remark. The conclusion of Theorem 6.1 is stronger than that of Theorem 6.2 even for azimuthally symmetric potentials since Theorem 6.1 says, in particular, that for small $\lambda$, the ground state will have $m=0$ if $-\Delta+V$ has a discrete ground state. Theorem 6.2 does not imply this since no uniformity in $m$ is claimed.

We begin with a negative result which motivates the need for our rather involved arguments.

Theorem 6.3. Let $V \in L^{2}+L_{\epsilon}^{\infty}$ and let $H_{0}(\lambda)$ be as above ( $\lambda$ real). Then $H_{0}(\lambda)+V$ does not converge in norm resolvent sense to $H_{0}+V$.

Proof. Suppose to the contrary that $H(\lambda) \equiv H_{0}(\lambda)+V$ converges to $H \equiv H_{0}+V$ in norm resolvent sense. Let $E<$ inf $\sigma(H)$. Then by Theorem 2.3, $E<\inf \sigma(H(\lambda))$. Let $P_{N}$ be the operator of multiplication by the characteristic function of $\{\vec{x}|| V(\vec{x}) \mid \leq N\}$. Then $\left\|P_{N}\left[V(H(\lambda)-E)^{-1}-V(H-E)^{-1}\right]\right\| \leq N \|$ $(H(\lambda)-E)^{-1}-(H-E)^{-1} \| \rightarrow 0$ for each $N$. Moreover, by Theorem 2.3 again

$$
\left\|\left(1-P_{N}\right) V\left[(H(\lambda)-E)^{-1}-(H-E)^{-1}\right]\right\| \leq 2\left\|\left(1-P_{N}\right) V(H-E)^{-1}\right\| \rightarrow 0
$$

as $N \rightarrow \infty$ since $V(H-E)^{-1}$ is compact. Thus under the contrary hypothesis $V(H(\lambda)-E)^{-1} \rightarrow V(H-E)^{-1}$ in norm. It follows that $\left(1-V(H(\lambda)-E)^{-1}\right)^{-1} \rightarrow$ $\left(1-V(H-E)^{-1}\right)^{-1}$ in norm since $E \notin \sigma\left(H_{0}\right)$ and thus that $\left(H_{0}(\lambda)-E\right)^{-1}=$ $(H(\lambda)-E)^{-1}\left(1-V(H(\lambda)-E)^{-1}\right)^{-1} \rightarrow\left(H_{0}-E\right)^{-1}$.

Write $H_{0}(\lambda)=p_{z}^{2}+\tilde{H}_{0}(\lambda), H_{0}=p_{z}^{2}+\tilde{H}_{0}$, where $\tilde{H}_{0}(\lambda)$ and $\tilde{H}_{0}$ are " 2 dimensional operators". Diagonalizing $p_{z}$ by a Fourier transform, we have on $L^{2}\left(\mathbb{R}^{2}\right)$ $\otimes L^{2}(d k)$ :

$$
\alpha(\lambda) \equiv\left\|\left(k^{2}+\tilde{H}_{0}(\lambda)-E\right)^{-1}-\left(k^{2}+\tilde{H}_{0}-E\right)^{-1}\right\| \rightarrow 0 .
$$

By looking at functions $\phi \otimes \psi(k)$ with $\psi$ peaked near $k=0$, we see that $\left\|\left(\tilde{H}_{0}(\lambda)-E\right)^{-1}-\left(\tilde{H}_{0}-E\right)^{-1}\right\|_{L^{2}(\mathbb{R})^{2}} \leq \alpha(\lambda)$ so $\left(\tilde{H}_{0}(\lambda)-E\right)^{-1}$ converges to $\left(\tilde{H}_{0}-E\right)^{-1}$ in norm resolvent sense. Since, for $\lambda \neq 0, \tilde{H}_{0}(\lambda) \uparrow\left(L_{z}=m\right)$ has compact resolvent, while $\tilde{H}_{0} \uparrow\left(L_{z}=m\right)$ does not, this is clearly impossible. Thus, we have established by contradiction that $H_{0}(\lambda)+V$ does not converge to $H_{0}+V$ in norm resolvent sense.

LEMMA 6.4. (a) For $\lambda$ real and $E \mathbb{E}[0, \infty),\left(H_{0}(\lambda)-E\right)^{-1}$ converges strongly to $\left(H_{0}-E\right)^{-1}$ as $\lambda \rightarrow 0$.

(b) Let $h_{0, m}(\lambda)=H_{0}(\lambda) \uparrow\left(L_{z}=m\right)$. Let $|\arg \lambda| \leq \theta_{0}<\frac{\pi}{2}$ and $|\arg E|>2 \theta_{0}$. Then $\left(h_{0, m}(\lambda)-E\right)^{-1}$ converges strongly to $\left(h_{0, m}-E\right)^{-1}$ as $|\lambda| \rightarrow 0$.

Proof. $\left.\| H_{0}(\lambda)-H_{0}\right) \phi \| \rightarrow 0$ for any $\phi \in C_{0}^{\infty}$. Since $C_{0}^{\infty}$ is a core for $H_{0}$, $\left\|\left[\left(H_{0}(\lambda)-E\right)^{-1}-\left(H_{0}-E\right)^{-1}\right] \eta\right\| \rightarrow 0$ for a dense set of $\eta$ whenever we have a uniform bound on $\left\|\left(H_{0}(\lambda)-E\right)^{-1}\right\|$. This type of uniform bound also allows us to 
go from a dense set of $\eta$ to all $\eta$. In case (a), the required uniform bound comes from the fact that $H_{0}(\lambda)$ is self-adjoint and positive. In case (b) we note that with $\theta=\arg \lambda$

$$
e^{-i \theta} h_{0, m}(\lambda)=e^{-i \theta}(-\Delta)+e^{i \theta} \rho^{2}|\lambda|^{2} / 4-|\lambda| m
$$

is sectorial up to the constant $|\lambda| m$.

LEMMA 6.5. Let $\left(1+\rho^{2}\right) W$ be $H_{0}$-bounded. Then $W\left(H_{0}-E\right)^{-1} \rho^{2}$ is bounded.

$$
\text { Proof. } \begin{aligned}
W\left(H_{0}-E\right)^{-1} x^{2}= & W x^{2}\left(H_{0}-E\right)^{-1}+4 i(x W)\left(H_{0}-E\right)^{-2} p_{x} \\
& +2 W\left(H_{0}-E\right)^{-2}-8 W\left(H_{0}-E\right)^{-3} p_{x}^{2}
\end{aligned}
$$

by a straightforward calculation. Each term is bounded.

LEMMA 6.6. (a) Under the hypothesis of Theorem 6.1, $V\left(H_{0}(\lambda)-E\right)^{-1}$ converges in norm as $\lambda \rightarrow 0$ to $V\left(H_{0}-E\right)^{-1}$ for $E \notin[0, \infty)$.

(b) Under the hypothesis of Theorem 6.2., $V_{(m)}\left(h_{0, m}(\lambda)-E\right)^{-1}$ converges as $\lambda \rightarrow 0\left(|\arg \lambda| \leq \theta_{0}\right)$ in norm to $V_{(m)}\left(h_{0, m}-E\right)^{-1}$ for $|\arg E|>2 \theta_{0}$ where $V_{(m)}=$ $V \uparrow\left(L_{z}=m\right)$,

Proof. We prove (a). The proof of (b) is similar if one uses the quadratic estimates of [49]. Suppose that we show that $\left\|V_{1}\left(H_{0}(\lambda)-E\right)^{-1}-V_{1}\left(H_{0}-E\right)^{-1}\right\|$ $\rightarrow 0$ for any $V_{1}$ with $\left(1+\rho^{2}\right) V_{1} \in L^{2}$. Then, by a limiting argument we get the result for $V \in \rho^{-2} L^{2}+L_{\epsilon}^{\infty}$. Now with $\vec{a}_{0}=\frac{1}{2}(0,0,1) \times \vec{r}$

$$
\begin{gathered}
V_{1}\left(H_{0}(\lambda)-E\right)^{-1}-V_{1}\left(H_{0}-E\right)^{-1}=V_{1}\left(H_{0}-E\right)^{-1}\left[2 \lambda \vec{a}_{0} \cdot \vec{p}-\lambda^{2} a_{0}^{2}\right] . \\
\left(H_{0}(\lambda)-E\right)^{-1}=V_{1}\left(H_{0}-E\right)^{-1}\left[2 \lambda \vec{a}_{0} \cdot\left(\vec{p}-\lambda \vec{a}_{0}\right)+\lambda^{2} \vec{a}_{0}^{2}\right]\left(H_{0}(\lambda)-E\right)^{-1} .
\end{gathered}
$$

By Lemma 6.5,

$$
V_{1}\left(H_{0}-E\right)^{-1} \vec{a}_{0} \text { and } V_{1}\left(H_{0}-E\right)^{-1} \vec{a}_{0}^{2}
$$

are bounded while $\left(\vec{p}-\lambda \vec{a}_{0}\right)_{j}\left(H_{0}(\lambda)-E\right)^{-1}$ is bounded by an elementary estimate.

Proof of Theorems 6.1 and 6.2. We prove Theorem 6.1. The other is similar. Since $E \in \sigma\left(H_{0}+V\right) \backslash \sigma\left(H_{0}\right)$ if and only if $1+V\left(H_{0}-E\right)^{-1}$ is not invertible, and $V\left(H_{0}(\lambda)-E\right)^{-1} \rightarrow V\left(H_{0}-E\right)^{-1}$ in norm, we have that for any $E \notin$ $\sigma\left(H_{0}+V\right)$, the operator $1+V\left(H_{0}(\lambda)-E\right)^{-1}$ is invertible for $\lambda$ small. Moreover $\left(1+V\left(H_{0}(\lambda)-E\right)^{-1}\right)^{-1} \rightarrow\left(1+V\left(H_{0}-E\right)^{-1}\right)^{-1}$ in norm. Since $\left(H_{0}(\lambda)-E\right)^{-1} \rightarrow$ $\left(H_{0}-E\right)^{-1}$ strongly, we see that $\left(H_{0}(\lambda)+V-E\right)^{-1} \rightarrow\left(H_{0}+V-E\right)^{-1}$ strongly as $\lambda \rightarrow 0$. This proves condition (1) for stability.

By the above, for $E \notin \sigma\left(H_{0}+V\right), V\left(H_{0}(\lambda)+V-E\right)^{-1} \rightarrow V\left(H_{0}+V-E\right)^{-1}$ in norm and thus using $A V B-A^{\prime} V B^{\prime}=A\left(V B-V B^{\prime}\right)+\left(A V-A^{\prime} V\right) B^{\prime}$ we see that

$$
\left(H_{0}(\lambda)-E\right)^{-1} V\left(H_{0}(\lambda)+V-E\right)^{-1} \rightarrow\left(H_{0}-E\right)^{-1} V\left(H_{0}+V-E\right)^{-1}
$$


equivalently

$$
\left(H_{0}(\lambda)+V-E\right)^{-1}-\left(H_{0}(\lambda)-E\right)^{-1} \rightarrow\left(H_{0}+V-E\right)^{-1}-\left(H_{0}-E\right)^{-1}
$$

in norm. Integrating around the circle $\left|E-E_{0}\right|=\epsilon$, and using the analyticity of $\left(H_{0}-E\right)^{-1}$ and $\left(H_{0}(\lambda)-E\right)^{-1}$ inside, we obtain norm convergence of the projections and so condition 2 .

APPENDIX. A theorem of Lieb

In a preprint version of this paper, we conjectured the inequality (5.1) which was subsequently proven by E. H. Lieb. We are endebted to Prof. Lieb for encouraging us to give his proof here. THEOREM A.1. Let $\vec{a}=\frac{1}{2}\left(\vec{B}_{0} x \vec{r}\right)$ and let $V$ obey the hypotheses of
Theorem 2.5. Let

$$
H=-(\vec{\nabla}-i \vec{a})^{2}+V
$$

Then

$$
\text { inf } \operatorname{spec}(H) \leq\left|\vec{B}_{0}\right|+\text { inf } \operatorname{spec}(-\Delta+V)
$$

Proof. By adding $\epsilon r^{2}$ to $V$ and taking $\epsilon$ to zero, we can suppose, without loss, that $E_{0} \equiv$ inf spec $(-\Delta+V)$ is an eigenvalue. Let $g$ be the corresponding eigenvector. Also suppose that $\vec{B}_{0}=\left(0,0, B_{0}\right)$. For $\vec{c}=\left(c_{x}, c_{y}, 0\right)$, let

$$
\psi_{\vec{c}}(x, y)=\exp \left[-\frac{1}{4} B_{0}\left[\left(x-c_{x}\right)^{2}+\left(y-c_{y}\right)^{2}\right]+i \frac{B_{0}}{2}\left(c_{x} y-c_{y} x\right)\right] .
$$

Notice that $\psi_{\overrightarrow{0}}$ is the "vacuum" for $H_{0}(\vec{a})$ centered at $\overrightarrow{0}$ and that $\psi_{\vec{c}}=$ $e^{-i \vec{c} \cdot \vec{\rho}} \psi_{\overrightarrow{0}}$ is the vacuum|centered at $\vec{c}$ where $\vec{\rho}$ is the pseudomomentum of $\S 3$. Let $h_{\vec{c}}$ be the trial vector $g \psi_{\vec{c}}$. Then, by a simple direct calculation

$$
\left(h_{\vec{c}}, H h_{\vec{c}}\right)=\left(E_{0}+\left|B_{0}\right|\right)\left(h_{\vec{c}}, h_{\vec{c}}\right)+\alpha(\vec{c})
$$

where

$$
\begin{aligned}
\alpha(\vec{c}) & =-\int \nabla|g|^{2} \cdot\left[\bar{\psi}_{c}(\vec{\nabla}-i \vec{a}) \psi_{c}\right] d^{3} x \\
& =\int|g|^{2} \operatorname{div}\left[\bar{\psi}_{c}(\vec{\nabla}-i \vec{a}) \psi_{c}\right] d^{3} x
\end{aligned}
$$

by an integration by parts. By another calculation (or using the covariance relating $\rho$ and $\pi), \bar{\psi}_{\vec{c}}(\vec{\nabla}-i \vec{a}) \psi_{\vec{c}}$ is only a function of $\left(x-c_{x}, y-c_{y}\right)$. Thus the derivative with respect to $x$ can be changed to a derivative with respect to $c$. We see that $\alpha(\vec{c})$ is the divergence of a function which goes to zero at infinity since $\psi_{0}$ is a Gaussian. Thus, $\int \alpha(\tilde{c}) d c_{x} d c_{y}=0$. In particular, $\alpha(\vec{c}) \leq 0$ for some $\vec{c}$ and so (A.1) implies (5.1).

Note. In [70], Hogreve et al have made a conjecture which includes (5.1) as a special case. A more general special case is the following conjecture which 
places (5.1) in an interesting light: Let $\vec{\sigma}$ be the Pauli matrices and let $\tilde{H}(\vec{a})$ $=-\bar{\nabla}-i \vec{a})^{2}+V+\vec{\sigma} \cdot \vec{B}$ where $\vec{B}=\vec{\nabla} x \vec{a}$, and the operator is viewed as acting on $L^{2}\left(R^{3}, C^{2}\right)$. Then [70] conjectures the paramagnetic inequality

$$
\text { inf spec } \sigma(\tilde{H}(\vec{a})) \leq \inf \operatorname{spec}(-\Delta+V)
$$

for arbitrary $\vec{a}$.

\section{REFERENCES}

1. S. Agmon, Spectral properties of Schrödinger operators and scattering theory, Ann. Scuola Norm. Sup. Pisa II 2(1975), 151-218.

2. J. Aguilar ANd J. M. Combes, A clasis of analytic perturbations for one-body Schrödinger Hamiltonians, Comm. Math. Phys. 22(1971), 269-279.

3. J. E. AVRon AND I. W. Herbst, Spectral and scattering theory of Schrödinger operators related to the Stark affect, Comm. Math. Phys. 52(1977), 239-254.

4. J. E. Avron, I. W. Herbst, and B. Simon, The Zeeman effect revisited, Phys. Rev. Lett. 62A(1977), 214-216.

5. __ Formation of negative ions in magnetic fields, Phys. Rev. Lett. 39(1977), 1068.

6. S _ Separation of the center of mass in homogeneous magnetic fields (Schrödinger operators in magnetic fields II), Ann. Physics, to appear.

7. __ Schrödinger operators with magnetic fields. III. Atoms in magnetic fields, to be submitted to Comm. Math. Phys.

8. M. S. Birman AND M. Z. SolomjaK, On estimates of singular numbers of integral operators, III. Operators on unbounded domains, Vest. LSU 24(1969); Eng. translation Vest. LSU Math 2(1975), 9-27.

9 ._ Estimation of the singular values of integral operators, Ups. Mat. Nauk. 32(1977), 1784.

10. R. Blankenbeckler, M. L. Goldberger, and B. Simon, The bound states of weaklycoupled long-range one-dimensional quantum Hamiltonians, Ann. Physics 108(1977), 69-78.

11. J. M. COMbes, R. Schrader, AND R. SEILER, Classical bounds and limits for energy distributions of Hamiltonian operators in electromagnetic fields, Ann. Physics, to appear.

12. M. CWICKEL, Weak type estimates for singular values and the number of bound states of Schrödinger operators, Ann. of Math. 106(1977), 93-102.

13. E. B. DAviES, Properties of Green's functions of some Schrödinger operators, J. London Math. Soc. 7(1973), 473-491.

14. __ Scattering from infinite sheets, Proc. Cambridge Philos. Soc., to appear.

15. P. DEIFT AND B. Simon, On the decoupling of finite singularities from the question of asymptotic completeness in two-body quantum systems, J. Functional Analysis 23(1976), 218238.

16. J. Dollard, Asymptotic convergence and the Coulomb interaction, J. Math. Phys. 5(1967), 729-738.

17. J. Dоoв, Stochastic Processes, Wiley, New York, 1953.

18. V. Enss, A note on Hunziker's theorem, Comm. Math. Phys. 52(1977), 233-238.

19. W. FARIS, Essential self-adjointness of operators in ordered Hilbert space, Comm. Math. Phys. 30(1973), 23-34.

20. J. Glimm AND A. JAFFe, “Boson Quantum field models,'” in Mathematics of Contemporary Physics, ed. R. Streater, Academic Press, 1972, New York, pp. 72-144.

21. A. Grossman, "Momentum-like constants of motion," in Statistical Mechanics and Field Theory, ed. R. N. Sen and C. Weil, Halsted Press, 1972.

22. I. Herbst, Unitary equivalence of Stark Hamiltonians, Math. Zeit. 155(1977), 55-70.

23. H. Hess, R. Schrader ANd D. A. Uhlenbrock, Domination of semigroups and generalization of Kato's inequality, Duke Math. J. 44(1977), 893-904. 
24. L. HöRMANDER, The existence of wave operators in scattering theory, Math. Zeit. 146(1976), 69-91.

25. T. IKEBE AND T. KATO, Uniqueness of the self-adjoint extension of singular elliptic differential operators, Arch. Rational Mech. Anal. 9(1962), 77-92.

26. A. JAFFE, A $\lambda \phi^{4}$ cutoff field theory, Princeton University thesis, 1965, unpublished.

27. K. JöRGENS, Zur spectral theorie der Schrödinger operators, Math. Zeit. 96(1967), 355-372.

28. K. Jörgens ANd J. WeIdMANN, Spectral Properties of Hamiltonian Operators, Springer Math. Notes No. 313, 1973.

29. T. Kato, Perturbation Theory for Linear Operators, Springer, 1966.

30. M. Klaus, On the bound state of Schrödinger operators in one dimension, Ann. Physics 108(1977), 288-300.

31. S. KuroDA, On the existence and the unitarity property of the scattering operator, Nuovo Cimento, 12(1959), 431-454.

32. __, On a theorem of Green and Lanford, J. Math. and Phys. 3(1962), 933-935.

33. _ Scattering theory for differential operators, I, II, J. Math. Soc. Japan 25(1973), 75-104; 222-234.

34. R. LAVINE AND M. O'CARROLL, Ground state properties and lower bounds on energy levels of a particle in a uniform magnetic field and external potential, J. Math. Phys. 18(1977), 1908-1912.

35. E. H. LIEB, Bounds on the eigenvalues of the Laplace and Schrödinger operators, Bull. Amer. Math. Soc. 82(1976), 751-753 and in prep.

36. E. H. Lieb, B. Simon, And A. S. Wightman, Studies in Mathematical Physics, Princeton University Press, 1976.

37. E. H. Lieb AND H. ThirRing, Bound for the kinetic energy of fermions which proves the stability of matter, Phys. Rev. Lett. 35(1975), 687.

38. Inequalities for the moments of the eigenvalues of the Schrödinger Hamiltonian and their relation to Sobolev inequalities, in [36].

39. M. ReEd ANd B. Simon, Methods of Modern Mathematical Phsyics, Vol. II Fourier Analysis, Self-Adjointness, Academic Press, New York, 1975.

40. —_ Methods of Modern Mathematical Physics, Vol. III Scattering Theory, Academic Press, New York, 1978.

41. ___ Methods of Modern Mathematical Physics, Vol. IV Analysis of Operators, Academic Press, New York, 1978.

42. —_ The scattering of classical waves from inhomogeneous media, Math. Zeit., 155(1977), 163-180.

43. G. V. RosENBLJUM, The distribution of the discrete spectrum for singular differential operators, Dokl. Akad. Nauk. 202(1972), no. 5.

44. M. SCHECHTER, Spectra of Partial Differential Operators, North Holland, Amsterdam, 1971.

45. E Essential selfadjointness of the Schrödinger operator with magnetic vector potential, J. Functional Analysis 20(1975), 93-104.

46. _ Scattering theory for elliptic systems, J. Math. Soc. Japan 28(1976), 71-79.

47. —_ Cut off potentials and forms extensions, Letters in Math. Phys. 1(1976), 265-273.

48. E. SeIler AND B. Simon, Bounds in the Yukawa $a_{2}$ quantum field theory: upper bounds on the pressure, Hamiltonian bound and linear lower bound, Comm. Math. Phys. 45(1975), 94-114.

49. B. Simon, Coupling constant analyticity for the anharmonic oscillator, Ann. of Phys. 58(1970), 76-136.

50. —_ Schrödinger operators with singular magnetic vector potentials, Math. Zeit. 131(1973), 361-370.

51. __ Univeral diamegnetism of spinless bose systems, Phys. Rev. Lett. 36(1976), 804-806.

52. __ On the number of bound states of two-body Schrödinger operators, a review, in [36].

53. __ The bound state of weakly coupled Schrödinger operators in one and two dimensions, Ann. of Phys. 97(1976), 279-288. 
54. __ Analysis with weak trace ideals and the number of bound states of Schrödinger operators, Trans. Amer. Math. Soc. 224(1976), 367-380.

55. __ An abstract Kato's inequality for generators of positivity preserving semigroups, Ind. Math. Journal 26(1977), 1067-1073.

56. __ Geometric methods in multiparticle quantum systems, Comm. Math. Phys. 55(1977), 259-274.

57. __ Kato's inequality and the comparison of semigroups, J. Functional Analysis, to appear.

58. __ Lower semicontinuity of positive quadratic forms, Proc. Roy. Soc. Edinburgh, to appear.

59. ___ Lectures on Trace Ideal Methods, Cambridge University Press, to appear.

60. ___ Functional Integration and Quantum Physics, Academic Press, New York, exp. 1979.

61. B. Simon AND R. HoEgh-KRöHN, Hypercontractive semigroups and two dimensional selfcoupled Bose fields, J. Functional Analysis 9(1972), 121-180.

62. D. R. YAFEEV, A remark concerning the theory of scattering for a perturbed polyharmonic operator, Math. Notes 15 (1974), 260-265.

63. R. P. Feynman And A. HibBs, Quantum Mechanics and Path Integrals, McGraw Hill, Hightstown, New Jersey, 1965.

64. T. KAто, Remarks on Schrödinger operators with vector potentials, Int. Eqn. and Op. Th., to appear.

65. L. PITT, A compactness condition for linear operators in function spaces, submitted to J. Op. Th.

66. B. Simon, Maximal and minimal Schrödinger operators and forms, J. Op. Th., to appear.

67. E. B. DAvies, Eigenfunction expansions for singular Schrödinger operators, Arch. Rational Mech. Anal. 63(1977), 261-272.

68. I. W. HERBST AND A. SloAn, Perturbation of translation invariant positivity preserving semigroups in $L^{2}\left(R^{N}\right)$, Trans. Amer. Math. Soc. 236(1978), 325-360.

69. P. G. Dodds AND D. H. Fremlin, Compact operators in Banach lattices, submitted to J. Functional Analysis.

70. H. Hogreve, R. Schrader, And R. Seiler, A conjecture on the spinor functional determinant, Free University of Berlin preprint.

AVron: NRCN, BEERSHEVA, ISRAEL

Herbst: Department of Mathematics, University of Virginia, Charlottesville, VirGINIA 22901

Simon: Departments of Mathematics and Physics, Princeton University, Princeton, NEW JERSEY 08540 